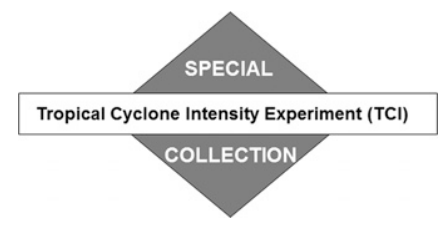

\title{
Improving Hurricane Analyses and Predictions with TCI, IFEX Field Campaign Observations, and CIMSS AMVs Using the Advanced Hybrid Data Assimilation System for HWRF. Part I: What is Missing to Capture the Rapid Intensification of Hurricane Patricia (2015) when HWRF is already Initialized with a More Realistic Analysis?
}

\author{
Xu Lu And Xuguang Wang \\ School of Meteorology, University of Oklahoma, Norman, Oklahoma
}

(Manuscript received 8 June 2018, in final form 1 February 2019)

\begin{abstract}
Assimilating inner-core observations collected from recent field campaign programs such as Tropical Cyclone Intensity (TCI) and Intensity Forecasting Experiment (IFEX) together with the enhanced atmospheric motion vectors (AMVs) produce realistic three-dimensional (3D) analyses using the newly developed GSI-based, continuously cycled, dual-resolution hybrid ensemble-variational data assimilation (DA) system for the Hurricane Weather Research and Forecasting (HWRF) Model for Hurricane Patricia (2015). However, more persistent surface wind maximum spindown is found in the intensity forecast initialized from the realistic analyses produced by the DA system but not from the unrealistic initial conditions produced through vortex modification. Diagnostics in this study reveal that the spindown issue is likely attributed to the deficient HWRF Model physics that are unable to maintain the realistic 3D structures from the DA analysis. The horizontal diffusion is too strong to maintain the realistically observed vertical oscillation of radial wind near the eyewall region. The vertical diffusion profile cannot produce a sufficiently strong secondary circulation connecting the realistically elevated upper-level outflow produced in the DA analysis. Further investigations with different model physics parameterizations demonstrate that spindown can be alleviated by modifying model physics parameterizations. In particular, a modified turbulent mixing parameterization scheme together with a reduced horizontal diffusion is found to significantly alleviate the spindown issue and to improve the intensity forecast. Additional experiments show that the peak-simulated intensity and rapid intensification rate can be further improved by increasing the model resolution. But the model resolution is not as important as model physics in the spindown alleviation.
\end{abstract}

\section{Introduction}

An accurate depiction of the tropical cyclone (TC) inner-core dynamic and thermodynamic structures is essential for the numerical prediction of TC intensity (Torn and Hakim 2009; Xiao et al. 2009; Zhang et al. 2009, 2011; Li et al. 2012; Weng and Zhang 2012; Lu et al. 2017a,b). However, TCs spend most of their lifetime over the open ocean, where high-resolution in situ innercore observations are often lacking. Although satellites can cover vast areas over the ocean, effective utilization of all-sky satellite radiance observations in the cloudy and rainy regions, such as the eyewall and spiral rainbands, is still immature (Bauer et al. 2010, 2011; Yang et al. 2016; Zhang et al. 2016; Zhu et al. 2018; Geer et al. 2018).

Corresponding author: Xuguang Wang, xuguang.wang@ou.edu
Because of the lack of high-resolution inner-core observations, early NWP studies usually initialized the TC predictions using a bogus vortex based on limited vortex information [e.g., radius of maximum wind (RMW), maximum wind speed (Vmax), and minimum sea level pressure (MSLP)] (Thu and Krishnamurti 1992; Kurihara et al. 1995, 1998, Liu et al. 2000, 2006; $\mathrm{Pu}$ and Braun 2001; Tallapragada et al. 2014). In the National Oceanic and Atmospheric Administration (NOAA) operational Hurricane Weather Research and Forecasting system (HWRF), vortex initialization (VI) contains two components: vortex relocation (VR) and vortex modification (VM), where VR corrects the storm location and VM modifies the storm intensity and size. Details can be found in Liu et al. $(2000,2006)$ and Tallapragada et al. (2015). Briefly, VR extracts the vortex from the background HWRF forecast. VM is then performed on the extracted vortex before it is 
placed back. During VM, the vortex size is first modified based on the RMW and the 34-kt $\left(17.5 \mathrm{~m} \mathrm{~s}^{-1}\right)$ wind radius. Then, the axisymmetric component of the vortex is rebalanced, and a historic axisymmetric composite storm is added to the vortex to adjust the storm intensity. The added composite storm is from a HWRF simulation of a real storm in 2007 (Tallapragada et al. 2015). However, numerous observations and studies have suggested that asymmetric dynamics can play important roles in the TC intensity prediction, especially during the intensification phase (Braun et al. 2006; Schubert et al. 1999; Reasor et al. 2004; Montgomery et al. 2002; Smith et al. 2008; Persing and Montgomery 2003; Reasor et al. 2000; Cram et al. 2007; Van Sang et al. 2008; Nguyen et al. 2011; Montgomery and Smith 2014; Persing et al. 2013). For example, the rapidly intensifying (RI) TCs are usually accompanied with asymmetric convective bursts (Montgomery et al. 2006; Fierro and Reisner 2011; Chen and Zhang 2013; Rogers et al. 2015; Susca-Lopata et al. 2015; Guimond et al. 2016). Therefore, more realistic inner-core initial conditions are required for TC predictions rather than the axisymmetric storms generated through VM. More recent studies suggested that efficient inner-core data assimilation (DA) can be a better initialization approach than VM when inner-core observations are available (Torn and Hakim 2009; Xiao et al. 2009; Zhang et al. 2009, 2011; Li et al. 2012; Weng and Zhang 2012; Aksoy et al. 2013; Schwartz et al. 2013; Lu et al. 2017a,b).

In recent years, unique opportunities to sample TC inner-core structures and near-core environments have been provided by different field campaigns such as the Hurricane Intensity Forecasting Experiment (IFEX) supported by NOAA (Rogers et al. 2013) and the Tropical Cyclone Intensity (TCI) Experiment supported by the Office of Naval Research (ONR) (Doyle et al. 2017). Airborne instruments from aircraft from these field campaigns can provide valuable high-resolution inner- and near-core information for TCs. Examples include Doppler radar observations collected by the NOAA WP-3D/G-IV aircraft during the IFEX field campaign, and dropsondes released by the WB-57 aircraft during the TCI field campaign. Together with the enhanced high-resolution atmospheric motion vector (AMV) wind observations provided by the Cooperative Institute for Meteorological Satellite Studies (CIMSS; Velden et al. 2017), an unprecedentedly thorough three-dimensional picture of TCs can be depicted including the inner-core, the environment, the outflow layer, and the low-level inflow regions. More specific information regarding these observations will be presented in Lu and Wang (2018, manuscript submitted to Mon. Wea. Rev., hereafter Part II).

A cycled, dual-resolution hybrid ensemble Kalman filter-variational (EnKF-Var) DA system based on the
National Weather Service operational DA system: Gridpoint Statistical Interpolation (GSI) was recently operationally implemented since 2017 . The approach adopted by this system is based on $\mathrm{Lu}$ et al. $(2017 \mathrm{a}, \mathrm{b})$. Lu et al. (2017a,b) demonstrated that the self-consistent ensemble covariance in this DA system was able to properly assimilate inner-core observations, which contributed to the improved intensity forecast. It is therefore expected that the three-dimensional (3D) analysis produced by this DA system ingesting the abundant observations provided by IFEX, TCI, and CIMMS AMVs mentioned above would be more realistic than the VM analysis.

One challenge associated with the intensity forecast by HWRF is the "spindown" issue (Bernardet et al. 2015; Zhou et al. 2015a,b; Pu et al. 2016; Tong et al. 2018). Following these early studies, spindown is defined as a significant vortex weakening [e.g., Vmax drop greater than $5 \mathrm{~m} \mathrm{~s}^{-1}$ $(6 \mathrm{~h})^{-1}$ ] during the first $\sim 6-12 \mathrm{~h}$ of the model forecast (Bernardet et al. 2015; Zhou et al. 2015a,b; Tong et al. 2018). This definition of spindown is used for the rest of the paper. Such a spindown issue sometimes jeopardizes the intensity forecasts especially for strong hurricanes during the intensification period. Most early studies attributed the spindown issue to the imbalanced, incomplete, or unrealistic initial analysis (Bernardet et al. 2015; Zhou et al. 2015b; Pu et al. 2016). However, as discussed in this paper, although a more realistic initial condition was generated for Hurricane Patricia (2015) using the field campaign observations and the GSI hybrid DA system compared to the VM analysis, more significant spindown occurs during the subsequent prediction initialized by the DA analysis than the VM analysis. This more significant spindown issue with a better DA analysis was also consistently found in Tong et al. (2018) with a larger sample size. The primary objective of this study is to investigate the reason behind it.

Some other studies (Vukicevic et al. 2013; Tong et al. 2018) indicated that the spindown issue is also likely be caused by the insufficient model physics, especially for the boundary layer physics. But no prominent evidence was found to support this hypothesis. As discussed later in the paper, our experiments suggest that the spindown is largely due to the deficiency associated with the HWRF Model physics schemes. Previous studies suggested that simulated TCs can be highly influenced by the turbulent diffusion processes (Emanuel 1997; Bryan and Rotunno 2009a,b; Bryan 2012; Rotunno and Bryan 2012; Zhang and Montgomery 2012; Gopalakrishnan et al. 2013; Montgomery and Smith 2014; Zhang and Marks 2015; Zhang et al. 2015). While a typical picture of TC secondary circulation in the eyewall region is an in-up-out cell (Montgomery and Smith 2014), some studies (Stern and Nolan 2011; Stern et al. 2014, 2017) suggested that 
there can be oscillating inflow and outflow in the vertical direction between the typical boundary layer inflow and the upper-level outflow in both observations and model simulations. Bryan and Rotunno (2009a) provides an analytical solution showing that these vertical oscillations of inflow and outflow in such TCs can be a result of unbalanced flow effects, in which the vertical subgradient and supergradient oscillation plays a key role instead of the gradient-wind balance in the eyewall region. However, these unbalanced flow effects can be damped through strong radial diffusion in the models (Bryan and Rotunno 2009a). Therefore, in this study, experiments are conducted to understand if the horizontal diffusion parameterization like the horizontal mixing length scale $L_{h}$ (a key parameter for horizontal diffusion configuration in the HWRF Model) is too large. The overly large horizontal diffusion may spuriously damp the realistically captured TC structures by DA analyses, which therefore results in spindown during the prediction of Hurricane Patricia (2015).

In addition to the horizontal diffusion, previous studies also suggested that the vertical diffusion can play a key role in the TC intensification process in HWRF. For example, Gopalakrishnan et al. (2013), Zhu et al. (2014), and Zhang et al. (2015) showed that the value of vertical diffusivity $K_{m}$ is important for the simulated maximum intensity in both the idealized and the operational HWRF. On the other hand, Bryan and Rotunno (2009b) found in the axisymmetric model that the maximum intensity of storms is insensitive to vertical diffusivity. Zhu et al. (2018) found that there was an unrealistic discontinuity of vertical diffusion near the boundary layer top in the HWRF planetary boundary layer (PBL) scheme applied in the HWRF Model (e.g., Fig. 1). This parameterization of vertical diffusivity $K_{m}$ was originally designed under a clear-sky assumption where the free atmosphere has little diffusion. Therefore, $K_{m}$ is always set to zero at the PBL top and the $K_{m}$ above the PBL is always following the clear-sky profiles in the HWRF PBL scheme. But this assumption is not suitable for the deep convection, such as the eyewall or spiral rainbands, where in-cloud turbulence creates large mixing above the PBL. Zhu et al. (2018) proposed a modified turbulent mixing parameterization scheme that replaces the boundary layer top with a "turbulent layer" top ${ }^{1}$ when calculating vertical diffusivity (e.g.,

\footnotetext{
${ }^{1}$ The turbulent layer is currently defined as the updraft greater than a certain critical value (e.g. $0.4 \mathrm{~m} \mathrm{~s}^{-1}$ in this study) above the boundary layer height. Therefore, in the non-deep-convection zone, the vertical diffusivity profile in the modified PBL scheme is identical to the original PBL scheme.
}

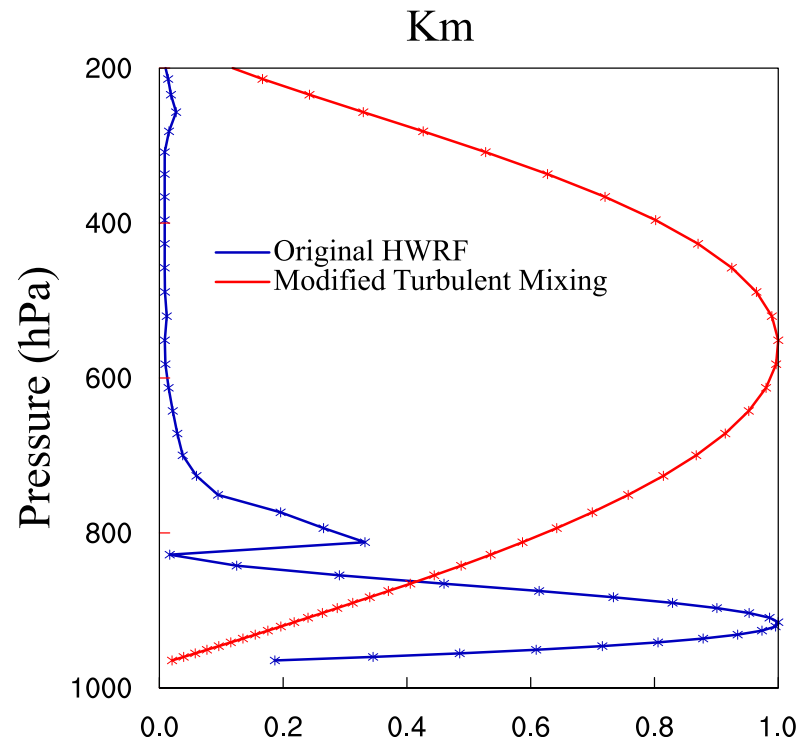

FIG. 1. An example of the normalized vertical diffusivity profile for the original HWRF (blue) and modified turbulent mixing (red) PBL parameterization scheme. These profiles are for the eyewall.

Fig. 1). Although the in-cloud mixing is further considered in this new scheme, the modified vertical diffusivity is still calculated using the empirical equations with slight adaptions. As a result, the level of the peak diffusivity is elevated from inside the PBL to the midlevel (e.g., $500 \mathrm{hPa}$ in Fig. 1) and the magnitude of the peak vertical diffusivity is larger (not shown). Although the modified profile still requires further evaluation given lack of direct observations, in a recent study by Zhu et al. (2018), this modified turbulent mixing parameterization significantly improves the intensity forecast for TCs. Therefore, in this study, the impacts of the newly proposed modified turbulent mixing parameterization scheme together with the sensitivity to $L_{h}$ are explored with the analysis produced from the advanced DA system to investigate the spindown issue. Detailed diagnostics are performed to explore how and why the deficiencies of the model physics parameterizations can contribute to the spindown issue.

Patricia (2015) was a category 5 hurricane that formed in the east Pacific on 20 October and made landfall along the southwestern coast of Mexico around 2300 UTC 23 October 2015 (Kimberlain et al. 2016). Many have claimed that this is the strongest observed $\mathrm{TC}$ in the east Pacific, with a maximum surface wind speed of $95.2 \mathrm{~m} \mathrm{~s}^{-1}$ (185 kt; Rogers et al. 2017). But most of the operational centers failed to forecast the RI and the strong peak intensity (Qin and Zhang 2018). In this study, experiments are carried out at 1800 UTC 22 October 2015 for the third TCI mission that sampled 


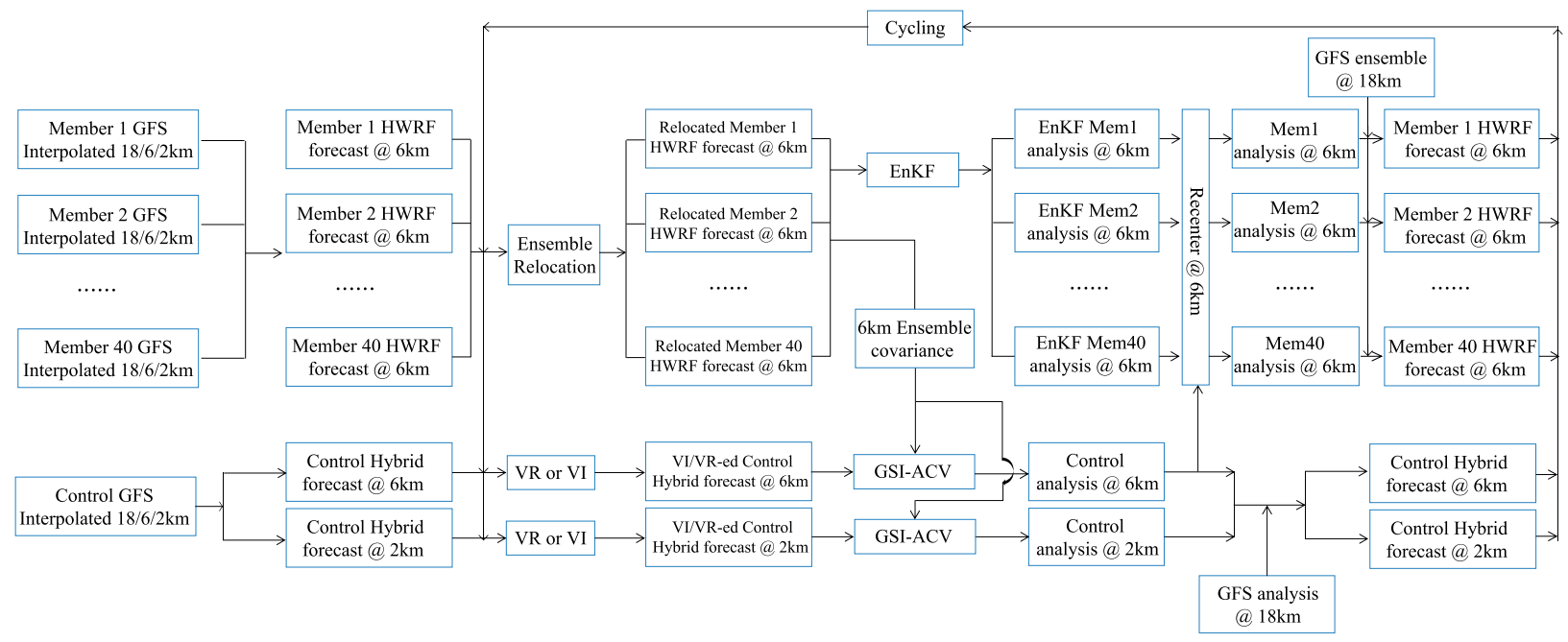

FIG. 2. Flowchart of the GSI-based EnVar hybrid DA system for HWRF [adapted and upgraded from Lu et al. (2017b)]. VI is a combination of VM and VR.

the RI phase of Patricia. A detailed description of the data collected by TCI is documented in Part II.

Considering the very small size of Patricia (smallest RMW recorded during Patricia's lifetime is about $9 \mathrm{~km}$ ), the 2-km model grid spacing configuration may not be enough to resolve the convective-scale features well in this case. Thus, in addition to investigating the issues associated with the model physics, experiments are also performed in this study to investigate the impact of model resolution on the intensity forecast and the spindown issue.

As the first part of a two-part study, we first describe the DA system, the model, and the experiment design in section 2. Then, section 3 shows the comparisons between VM and DA analyses and their subsequent forecast. As part of the comparison, investigations are carried out to understand why the more persistent TC spindown exists with the more realistic DA analysis but not the unrealistic VM analysis. Section 4 explores the impacts of model PBL physics parameterization changes on the spindown issue. Section 5 describes the impact of model resolution on the TC forecast and spindown issue and section 6 summarizes and concludes the results.

\section{Methodology, data, and experiment design}

\section{a. System description}

Following Lu et al. (2017b), the newly developed GSIbased, continuously cycled, dual-resolution hybrid ensemblevariational (EnVar) DA system for HWRF is used in this study with some upgrades. To be consistent with the model resolution update in the 2015 operational
HWRF (Tallapragada et al. 2015), a 18/6/2-km grid spacing configuration is used in this study instead of the previous $27 / 9 / 3-\mathrm{km}$ grid spacing in $\mathrm{Lu}$ et al. (2017b). Figure 2 shows the flowchart of this upgraded hybrid DA system with adaptations from $\mathrm{Lu}$ et al. (2017b). For consistency, the following descriptions parallel that of Lu et al. (2017b) with adaptations and simplifications.

At the initial cycle of a storm, a $40-$ member $18 / 6-\mathrm{km}$ doubly nested HWRF ensemble and a single deterministic 18/6/2-km triply nested HWRF control are initialized from the Global Forecast System (GFS) ensemble and control analyses, respectively. These analyses are obtained from the National Centers for Environmental Prediction (NCEP) operational GFS hybrid DA system (Wang et al. 2013; Wang and Lei 2014). Then, VR is performed on both ensemble and control forecasts and VM is only performed on the control forecast and only when the inner-core observations are unavailable. The details on how to perform VR and VM can be found in Lu et al. (2017b) section 2e. These updated control and ensemble forecasts will be used as the background for the next DA. To solve the nonoverlapping domain issue associated with the moving nests for the hybrid DA, the newly developed directed moving nest strategy is adopted during cycled ensemble and control forecasts following Lu et al. (2017b).

At the DA stage, a 40-member analysis on the 6-km grid is first produced by EnKF (ensemble Kalman filter) based on the relocated HWRF background ensemble forecast. Next, the modified control analysis on the 2- and 6-km domains is produced by the hybrid 3DEnVar using the same HWRF ensemble and 
the augmented control vector (ACV) method (e.g., Wang et al. 2007; Wang 2010; Wang et al. 2013). Then the EnKF mean on the 6-km grid is replaced with the 6-km hybrid analysis to recenter the EnKF ensemble. During recentering, the ensemble mean is replaced by the hybrid control analysis. After recentering, the outermost HWRF domain on the 18-km grid is replaced by the GFS control and ensemble analyses. Similar recentering is adopted for the global hybrid DA system (Wang et al. 2013).

After the DA stage, the updated analyses are used to produce background forecasts for the next DA cycle. For example, a 9-h deterministic forecast is initialized from the hybrid analysis on the 18/6/2-km grid, and a 6-h 40-member ensemble background forecast is initialized from the EnKF analysis on the $18 / 6-\mathrm{km}$ grids. The directed moving nest strategy is also applied during the forecasts following Lu et al. 2017b, and VR and VM procedures are conducted before applying the next DA stage. Meanwhile, an independent single deterministic 120 -h free forecast is initialized from the hybrid analysis on the 18/6/2-km grid using HWRF's original vortex-following strategy. The above DA and forecast cycles are continuously repeated until the end of the storm. This cycled, self-consistent hybrid DA system was not implemented to operational HWRF until 2017. However, the same operational HWRF Model in 2015 is used as a baseline in this study.

\section{b. HWRF Model configuration}

The HWRF Model was developed by the Environmental Modeling Center (EMC) in collaboration with the Geophysical Fluid Dynamics Laboratory (GFDL) and the University of Rhode Island (URI) based on the Weather Research and Forecasting (WRF) Model infrastructure and NonHydrostatic Mesoscale Model (NMM) dynamic core (Tallapragada et al. 2015). As mentioned in the previous section, the operational HWRF has experienced a major update in the model grid spacing from $27 / 9 / 3 \mathrm{~km}$ to $18 / 6 / 2 \mathrm{~km}$ in 2015 . Correspondingly, a horizontal grid spacing of $0.135 \%$ $0.045^{\circ} / 0.015^{\circ}$ (approximately $18 / 6 / 2 \mathrm{~km}$ ) for the outermost/intermediate/innermost domains are used for the newly developed hybrid DA system in this study, which is similar to that used in the 2015 operational HWRF. The outermost, intermediate, and innermost domains are configured with $288^{\circ} \times 576^{\circ}$ (roughly $\left.80^{\circ} \times 80^{\circ}\right), 304^{\circ} \times 604^{\circ}\left(\right.$ roughly $\left.30^{\circ} \times 30^{\circ}\right)$ and $265^{\circ} \times$ $472^{\circ}$ (roughly $7^{\circ} \times 7^{\circ}$ ) horizontal grid points, respectively [domain size similar to Fig. 3 in $\mathrm{Lu}$ et al. (2017b); not shown]. There are 61 vertical levels and the model top is at $2 \mathrm{hPa}$ following the operational HWRF. The physics parameterization schemes used in this study follows those used in the 2015 operational HWRF (details can be found in Table 1).

\section{c. Experiment design}

To understand why TC spindown exists with the more realistic DA analysis and how the model PBL physics parameterization and model resolution can impact the spindown and intensity forecasts, eight experiments denoted as "NoDA-warm," "NoDA," "VM," "DA," "DA-Hi," "DA-HD," "DA-HDVD," and "DA-HDVDHi" are conducted (see descriptions in Table 1). The analysis time of interest is 1800 UTC 22 October 2015. Details for each experiment are described as below:

NoDA-warm is a 6-h "nonstop" free forecast initialized from the 1200 UTC DA analyses on 22 October 2015. Seven cycles of DA and forecasts were performed before hand starting from 1800 UTC 20 October 2015 when Patricia became a tropical depression EP20 using the cycled DA system as described in Fig. 2 and section 2a. During these DA cycles, the same observations from the operational HWRF (Tallapragada et al. 2015) are assimilated as listed in Table 1.

In the NoDA experiment, the forecast is initialized by the relocated control background valid at 1800 UTC 22 October 2015. Compared to NoDA-warm which is more like a "restart" run, NoDA zeroes out the vertical velocities and hydrometeors, performs vortex relocation and replaces the outermost domain with the GFS analysis. Comparison between NoDA and NoDA-warm will reveal if these procedures typically done before each DA update can contribute to the spindown issue.

Experiments VM and DA perform purely VM and DA, respectively, based on the relocated background from NoDA. Specifically, DA assimilates the inner-core and near inner-core observations from IFEX, TCI field campaigns and CIMSS AMV in addition to the operational observations upon NoDA (specific observation types are listed in Table 1 of this paper and details of each type of observations are introduced in Part II). The satellite radiances from the operational observations are only assimilated in the intermediate domain following Lu et al. (2017b) and the operational HWRF configuration (Tallapragada et al. 2015). Slightly adjusted from Lu et al. (2017a,b), the horizontal and vertical localizations used in this study are configured to be $150-\mathrm{km} /$ $180-\mathrm{km}$ and $-0.46 /-0.46$ scale-height recursive filter covariance localization length scale (Barker et al. 2004; Wang et al. 2008; Pan et al. 2014) for the innermost/ intermediate domains, respectively. Full ensemble error covariance is used for both DA domains in this study following the configurations in Lu et al. (2017a,b). Intercomparison between NoDA, VM and DA can help 


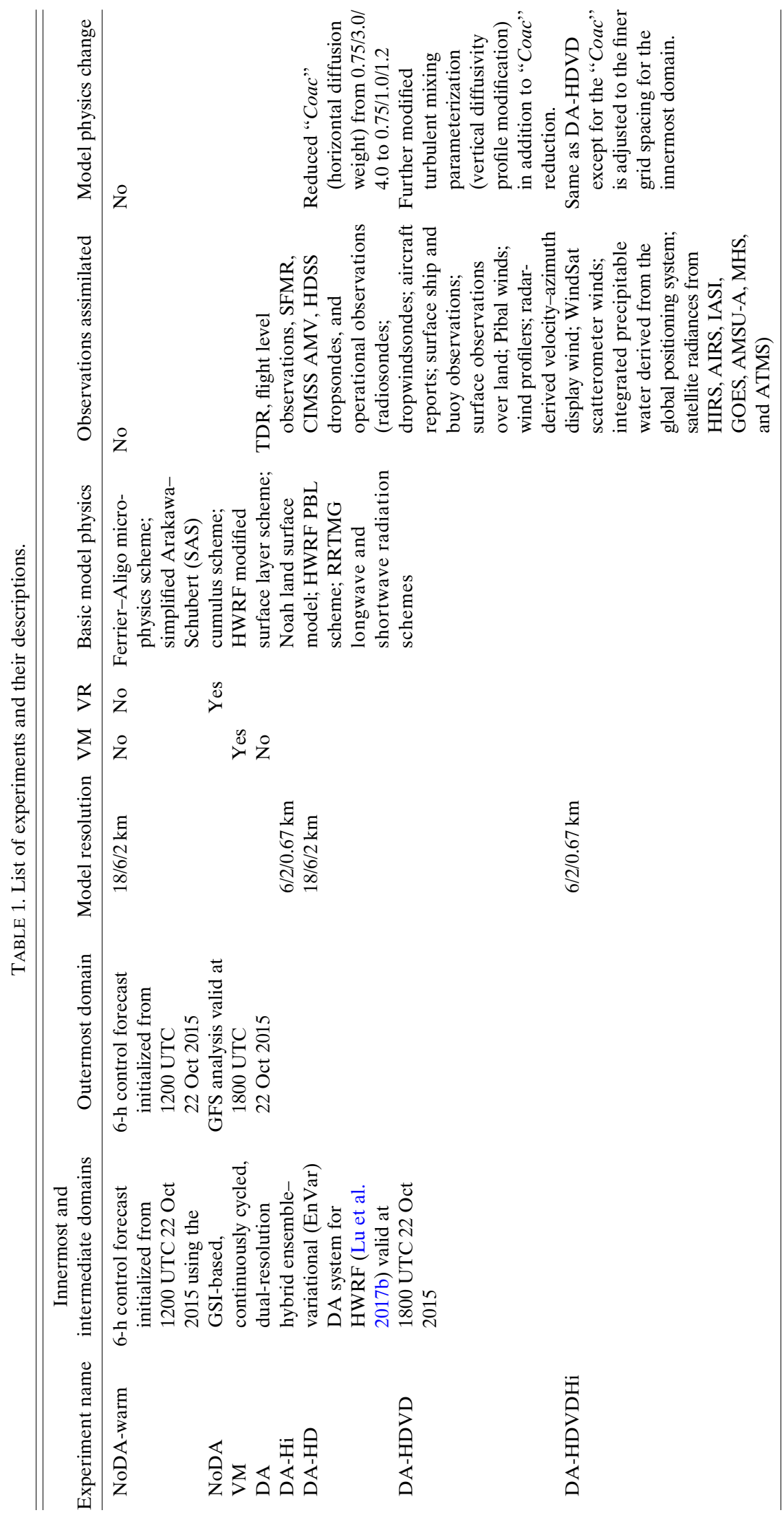


reveal why the more persistent TC spindown occurs in the realistic DA but not in the unrealistic VM.

DA-Hi is a high-resolution (6/2/0.67-km grid spacing) forecast based on DA. Because of the constraints in the HWRF Model infrastructure, current HWRF Model does not support the quadruple nest domain configuration. Therefore, to investigate the model resolution impact while maintaining the benefits of the continuously cycled hybrid DA system, we utilize the relocation package from the operational HWRF (Liu et al. $2000,2006)$ to downscale the analysis produced by the hybrid DA (2-km resolution) onto a higher-resolution domain $(0.67-\mathrm{km}$ resolution). Specifically, the analyses from $18 / 6 / 2-\mathrm{km}$ grid spacing domains are first merged into one combined domain (3-km resolution). Next, the combined domain is interpolated onto finer $6 / 2 / 0.67-\mathrm{km}$ grid spacing domains. Then, a 120 -h free forecast is launched based on these interpolated analyses. The goal of this high-resolution experiment is to investigate the impact of model resolution on both the spindown issue and the TC peak intensity forecast.

DA-HD is similar to DA except using a reduced "Coac" (horizontal diffusion weighting factor) physics parameterization configuration from 0.75/3.0/ 4.0 to $0.75 / 1.0 / 1.2$ for the outermost/intermediate/ innermost domains, respectively. The "Coac" controls the magnitude of $L_{h}$. The larger the "Coac", the more the horizontal mixing. Different "Coac" values in different domains are due to the model grid spacing dependency of the horizontal eddy diffusivity (Janjić 1990; Zhang et al. 2018). According to Zhang and Marks (2015), the configuration of $0.75 / 3.0 / 4.0$ was designed for large horizontal grid spacing. Such a configuration is equivalent to the $L_{h}$ of $1900-\mathrm{m}$ (Zhang et al. 2018). As a result, in the 2016 operational HWRF, the "Coac" is reduced to 0.75/1.0/1.2, which gives an estimate of $L_{h}$ at about 800-m. Zhang et al. (2018) claimed this new set of values are more consistent with the observational estimate and the model resolution (Zhang and Montgomery 2012). This latter set of values is used in this study for the "DA-HD" experiment. To reduce the accumulated effect from altered diffusion over multiple cycles, the model physics are only modified $6 \mathrm{~h}$ prior the target DA cycle in this study.

Experiment DA-HDVD is based on DA-HD where a modified PBL scheme is further used to modify the vertical diffusion profile in the HWRF Model in addition to the "Coac" reduction. This modified PBL scheme is proposed by Zhu et al. (2018) and is discussed in section 1 . Intercomparison among DA, DA-HD, and DA-HDVD will reveal how the model PBL physics changes impact the TC spindown and intensity forecasts.
DA-HDVDHi is similar to DA-Hi except using the modified PBL physics including the reduced "Coac" and the modified PBL scheme. To be consistent with the increased model resolution, the "Coac" is also adjusted to the finer grid spacing for the innermost domain. Comparison between DA-HDVD and DA-HDVDHi will reveal the impact of model resolution on TC intensity forecast with the modified model physics. Intercomparison among DA, DA-Hi, DA-HDVD and DA-HDVDHi will reveal the relative contribution of model physics and model resolution on the spindown issue and on the peak intensity forecasts of Patricia.

\section{Why more persistent TC spindown exists with the more realistic analyses produced by DA?}

\section{a. Differences between VM and DA on TC analyses and forecasts}

The performances of VM and DA are first compared together with NoDA in this subsection to investigate their impacts on TC analyses and forecasts in the HWRF Model. The horizontal wind structures at different levels produced by the VM and DA analyses are first verified against the observations and the radar wind composite. Figure 3 shows the model-derived wind and the corresponding verifications at the surface and $3-\mathrm{km}$ height valid at 1800 UTC 22 October 2015. The surface verification is from the observations of SFMR (Stepped Frequency Microwave Radiometer) on board the NOAA WP-3D aircraft and the 3-km height verification is composited from the TDR radial velocity data provided by HRD (Gamache 2005; both observations can be obtained from HRD 2015). While the SFMR observations suggested a small size hurricane (RMW about $18 \mathrm{~km}$ ) with strong surface wind maximum (close to $60 \mathrm{~m} \mathrm{~s}^{-1}$; Fig. 3a) around the northeast of Patricia at this time, experiment NoDA produces a spuriously large hurricane (RMW about $42 \mathrm{~km}$ ) with much weaker surface wind maximum (about $41 \mathrm{~m} \mathrm{~s}^{-1}$ ) to the southeast (Fig. 3b). This spuriously large size is also found in the 3-km height verifications above the surface (Figs. 3g,h). Although the magnitude of wind maximum at the $3-\mathrm{km}$ height is comparable with the observations, the wind speed in the southwest of the storm in NoDA is too strong (Figs. 3g,h). Therefore, modifications such as VM or DA are necessary to improve this suboptimal initial condition.

The corresponding analyses produced by VM and DA at different heights are shown in Figs. 3c, 3d, 3i, and 3j. In the VM experiment, an axisymmetric composite vortex from the historic HWRF Model forecasts is added onto the background to enhance storm intensity (Tallapragada et al. 2015). As a result, although the value of surface wind 
a) SFMR@10 m 1739Z22

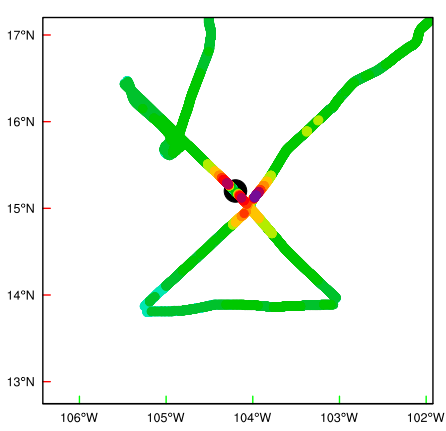

d) DA@10m 1800Z22

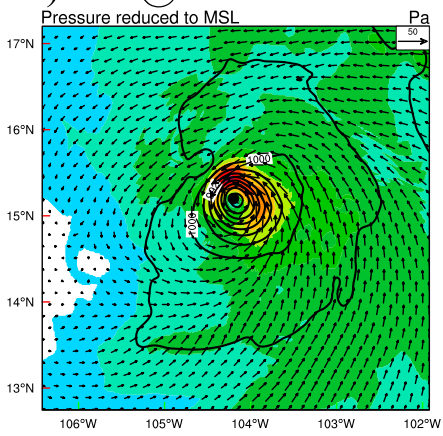

g)HRD@3km 1739Z22

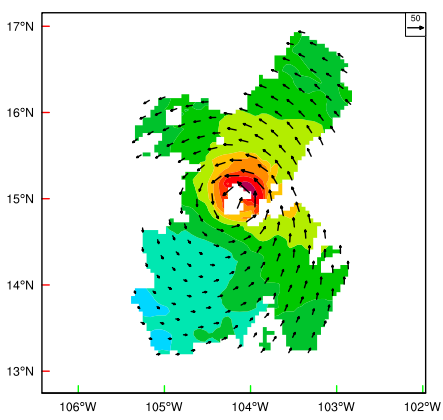

j) DA@3km 1800Z22

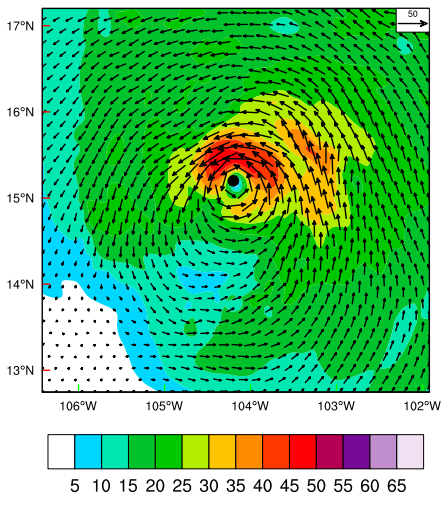

$\mathrm{m} / \mathrm{s}$ b) NoDA @10m 1800Z22

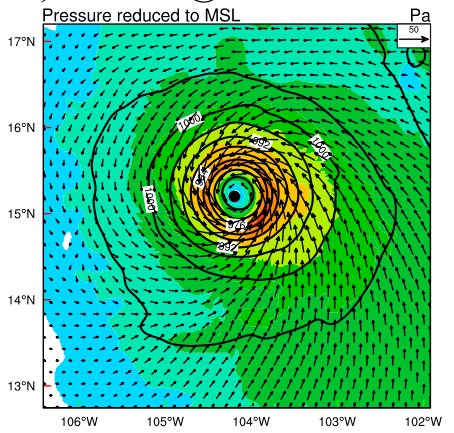

e) DA-HD@10m 1800Z22

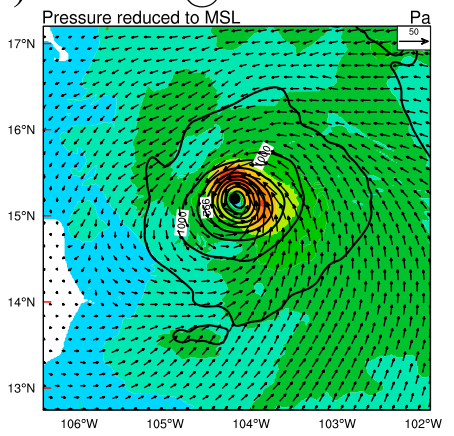

h) NoDA@3km 1800Z22

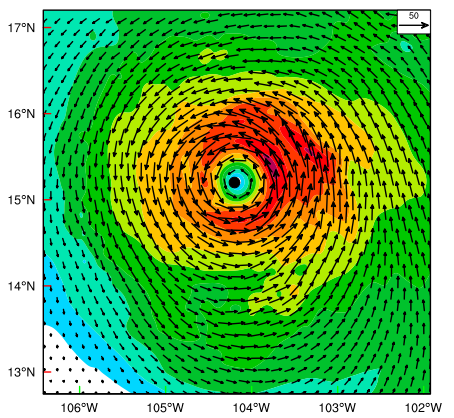

k) DA-HD@3km $1800 Z 22$ 1)

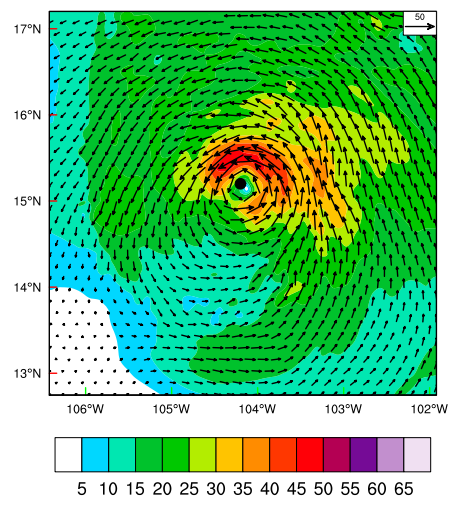

$\mathrm{m} / \mathrm{s}$ c) VM@10m 1800Z22

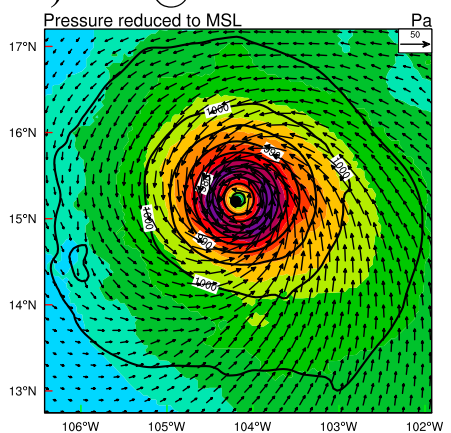

f) DA-HDVD @10m 1800Z22

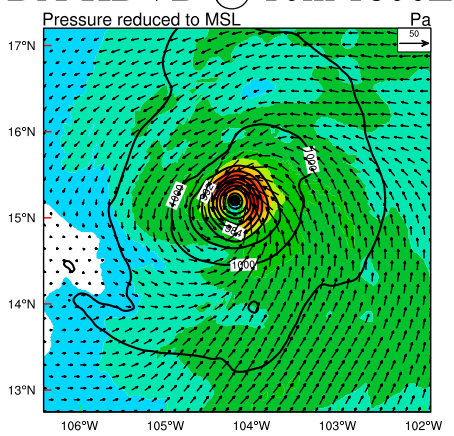

i) VM@3km $1800 Z 22$

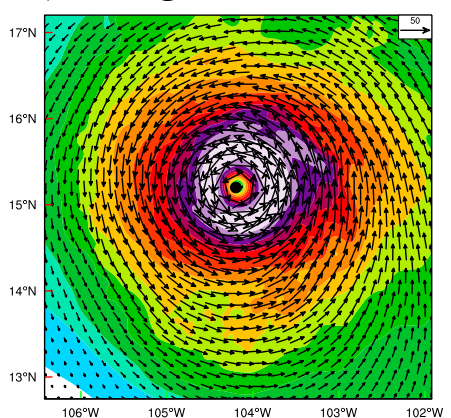

1) DA-HDVD@3km 1800Z22

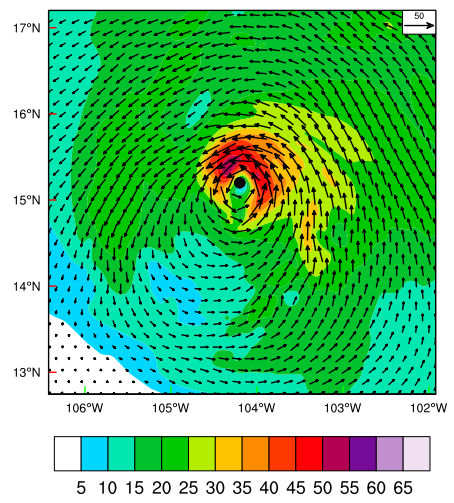

$\mathrm{m} / \mathrm{s}$

FIG. 3. Wind (shading and vectors, $\mathrm{m} \mathrm{s}^{-1}$ ) and pressure (contours, hPa) at 10-m height for (a) SFMR observations, (b) NoDA analysis, (c) VM analysis, (d) DA analysis, (e) DA-HD analysis, and (f) DA-HDVD analysis for the third TCI mission valid at 1800 UTC 22 Oct 2015. The black dot is the best track position from NHC. The corresponding wind (shading and vectors) at 3-km height for (g) HRD radar composite, (h) NoDA analysis, (i) VM analysis, (j) DA analysis, (k) DA-HD analysis, and (l) DA-HDVD analysis. Note the SFMR and HRD radar composite are centered around 1739 UTC 22 Oct 2015. There are no wind vectors in the SFMR observations in (a) and there are no pressure contours in both the SFMR and TDR observations [(a) and (g), respectively]. 
maximum in VM is modified and is now more consistent with the operational intensity estimate and the best track (Fig. 5a), the wind maximum above the surface becomes spuriously strong (e.g., Fig. 3i). Meanwhile, although the RMW of the VM analysis is reduced slightly due to the size modification, the size of the 34-kt wind radii is significantly increased after adding the strong axisymmetric composite. In other words, the primary circulation in VM becomes spuriously large and strong. In comparison with VM, experiment DA produces a significantly contracted vortex where both the RMW and 34-kt wind radii are more consistent with the SFMR observations (Fig. 3d). This storm size reduction is consistently found at different levels (e.g., Fig. 3j). Moreover, the spuriously strong wind flows in the southwest quadrant found in NoDA are reduced to be more comparable with the HRD radar composite. However, the wind maximum in DA is positioned to the north rather than the northeast of the storm. In addition, the magnitude of this wind maximum (about $52 \mathrm{~m} \mathrm{~s}^{-1}$ ) is still weaker than the SFMR observations at the surface. But overall, the general 3D TC inner-core structure produced by the DA experiment fits observations much better than either NoDA or VM. Further details on how assimilated observations from IFEX, TCI, and CIMSS can improve various aspects of the analysis of Patricia are presented in Part II.

To further diagnose the differences among NoDA, $\mathrm{VM}$, and DA, the gradient wind balance (GWB) relationship within each experiment is investigated. Following Smith et al. (2009), a net radial force (NRF) field defined as the difference between the local radial pressure gradient force and the sum of centrifugal force and Coriolis force is used to describe the GWB relationship. This NRF is calculated on the pressure coordinates following Pu et al. (2009):

$$
\mathrm{NRF}=-g \frac{\partial z}{\partial r}+\frac{v^{2}}{r}+f_{0} v
$$

where $z$ is the geopotential height, $r$ is the radial distance, $v$ is the tangential wind speed, and $g$ and $f_{0}$ are the constants of gravitational acceleration and Coriolis parameter, respectively. Therefore, the GWB is established when NRF equals 0 , and the flow is supergradient or subgradient when NRF is larger or smaller than 0 , respectively. The corresponding azimuthal mean NRF fields for each experiment in the radius-pressure cross section are shown in Fig. 4.

According to some previous studies, the boundary layer of an intensifying storm is found to be subgradient at outer radii and supergradient at inner radii (Smith et al. 2008, 2009). Figures 4a and 4c show that NoDA and DA are producing consistent boundary layer NRF fields with these theoretical studies. In the free atmosphere regions above the PBL, previous studies often assumed GWB and hydrostatic balance (Emanuel 1986, 1995). Consistently, Figs. $4 \mathrm{~d}$ and $4 \mathrm{f}$ indicate that the NRF fields in both NoDA and DA are almost 0, or GWB, outside the eyewall region above PBL. Additionally, as indicated in section 1, studies by Bryan and Rotunno (2009a,b) analytically showed that these hydrostatic and gradient-wind balances are violated near the eyewall region. They found unbalanced subgradient and supergradient oscillations associated with inflow/outflow oscillations in the vertical directions around the eyewall. Stern et al. $(2014,2017)$ showed evidence from simulations for these unbalanced oscillations in the eyewall with strong small-size storms like Patricia (2015). Moreover, Stern et al. (2017) suggested that the atypical midlevel wind speed maxima (centered around $6 \mathrm{~km}$ ) found in Patricia observations on 23 October were attributed to the unbalanced flow oscillations. Consistently, the vertical subgradient/supergradient oscillations are captured by the negative/positive oscillating NRF field around the eyewall in DA analyses above the PBL although it is difficult to verify this due to the lack of enough 3D pressure observations. Thus, only the verifications of the wind-related terms in the GWB equation (sum of centrifugal force and Coriolis force terms) against those calculated from the HRD radar composite are conducted. The results show that the DA analysis is much more consistent with the verifications than NoDA and VM (Figs. $4 \mathrm{~g}-\mathrm{j}$ ). In comparison with DA and NoDA, Figs. $4 \mathrm{~b}$ and $4 \mathrm{e}$ indicate that $\mathrm{VM}$ is producing a significantly positive NRF field throughout the PBL and above. The spurious, positive NRF, or supergradient, field in VM can be found far away from the eyewall even up to the $120-\mathrm{km}$ radii. This spurious and strong supergradient field in VM is a sign of initial vortex imbalance according to Pu et al. (2016).

In addition to the verifications of the TC structure analyses, the Vmax, MSLP and track forecasts initialized from these analyses are verified against the best track data in Fig. 5. Without any DA or VM, NoDA predicts an intensifying storm. However, the initial intensity in NoDA is too weak and the intensification rate is slower than best track after hour 6 (e.g., Figs. 5a,b). Consequently, the forecasted peak Vmax and MSLP in NoDA is about $32 \mathrm{~m} \mathrm{~s}^{-1}$ and $58 \mathrm{hPa}$ weaker than the best track, respectively. In addition, the track forecast from NoDA suggests an eastward bias at the early lead times before landfall (Fig. 5c). In comparison with NoDA, regardless of the unrealistically large, strong and supergradient imbalanced analyses shown earlier in this 


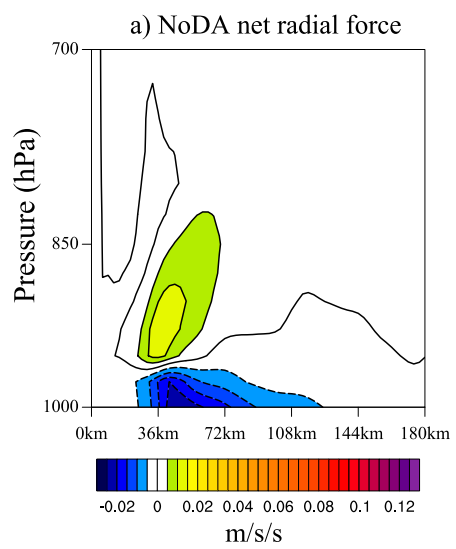

d) NoDA net radial force

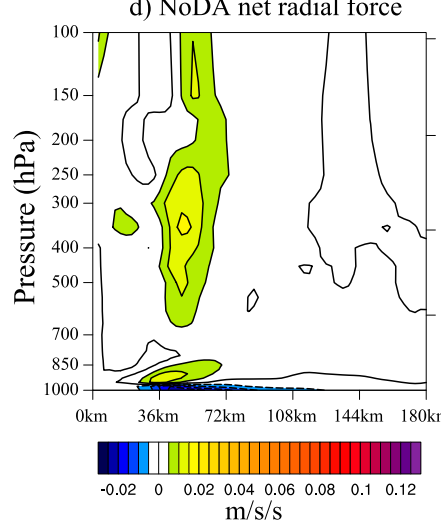

g) NoDA CenF + CorF

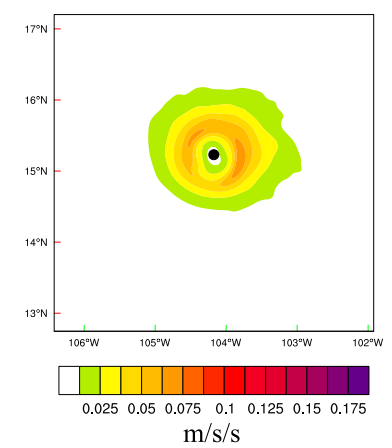

b) VM net radial force

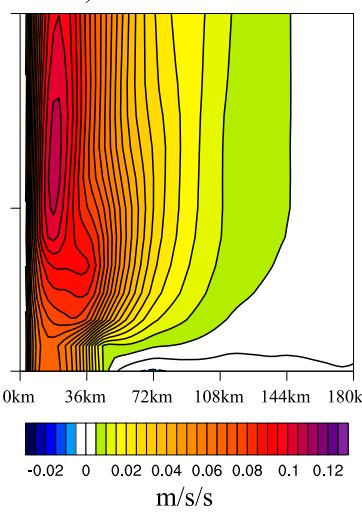

e) VM net radial force

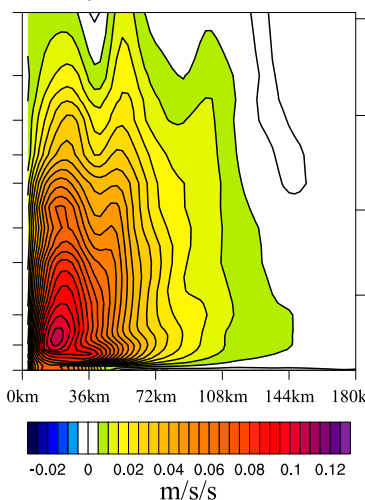

h) $\mathrm{VM} \mathrm{CenF}+\mathrm{CorF}$

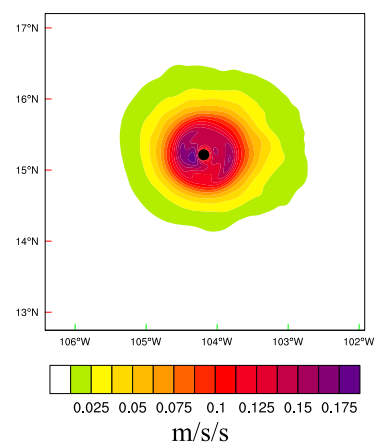

c) DA net radial force

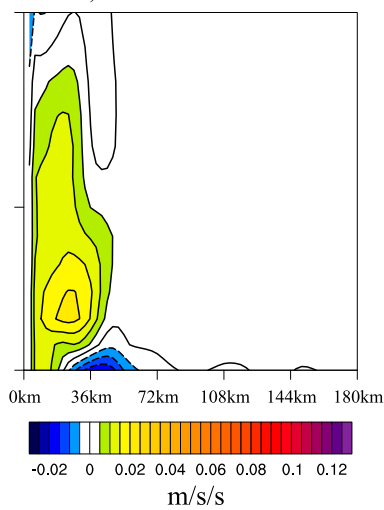

f) DA net radial force

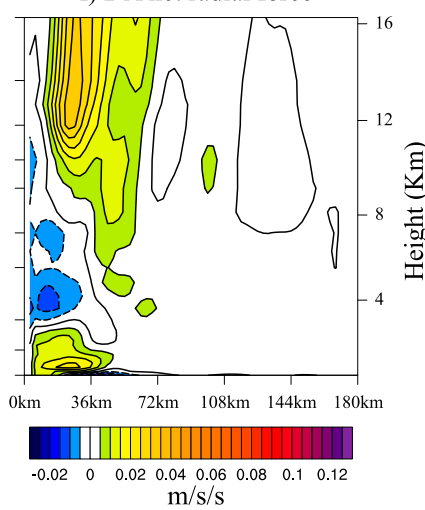

i) $\mathrm{DA}$ CenF + CorF

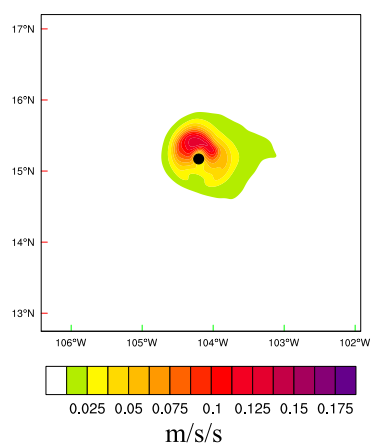

j) $\mathrm{HRD}$ CenF + CorF

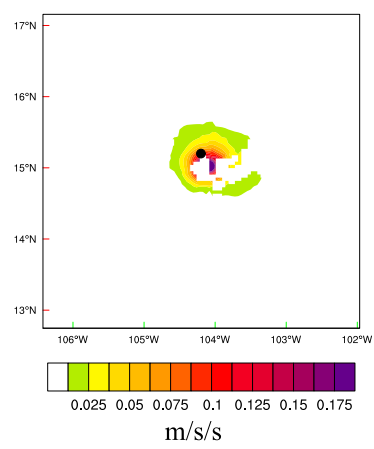

FIG. 4. Radius-height cross section of the isopleths of the azimuthal mean NRF ( $\mathrm{m} \mathrm{s}^{-1} \mathrm{~s}^{-1}$ ) for (a),(d) NoDA, (b),(e) VM, and (c),(f) DA analysis at 1800 UTC 22 Oct 2015. (a)-(c) A zoom-in plot for (d)-(f) below $700 \mathrm{hPa}$ and within 90-km radii. The sum of centrifugal force and Coriolis force $\left(\mathrm{m} \mathrm{s}^{-1} \mathrm{~s}^{-1}\right)$ at $1-\mathrm{km}$ height is also given for (g) NoDA, (h) VM, (i) DA, and (j) HRD radar composite.

subsection (Figs. 3c,i and Figs. 4b,e), VM apparently improves the Vmax and MSLP forecasts upon NoDA although the track forecast is only slightly improved. For example, the initial values of Vmax and MSLP in VM are more consistent with the best track due to the intensity modification (Figs. 5a,b). Additionally, the peak intensity in VM is also improved over NoDA where the peak values of Vmax and MSLP are now only $24.7 \mathrm{~m} \mathrm{~s}^{-1}$ and $39 \mathrm{hPa}$ weaker than the best track, respectively. Nevertheless, the intensification rate of VM is unreasonably slow and the timing of peak intensity in $\mathrm{VM}$ is shifted $6 \mathrm{~h}$ earlier as compared to the best track. In comparison with VM, although DA apparently improves the initial storm structures and the initial Vmax values upon NoDA, the intensity forecast from DA is degraded. In particular, significant Vmax spindown (about $13 \mathrm{~m} \mathrm{~s}^{-1}$ drop for the first $6 \mathrm{~h})$ happens in DA. As a result, the intensification in DA is delayed and the peak intensity is even weaker than NoDA. But regardless of the intensity forecast 
a) Vmax Forecasts

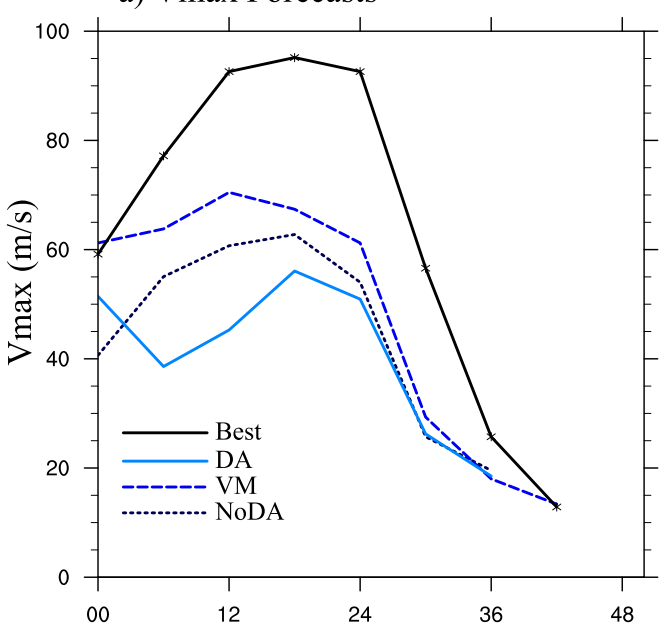

c) Track Forecasts

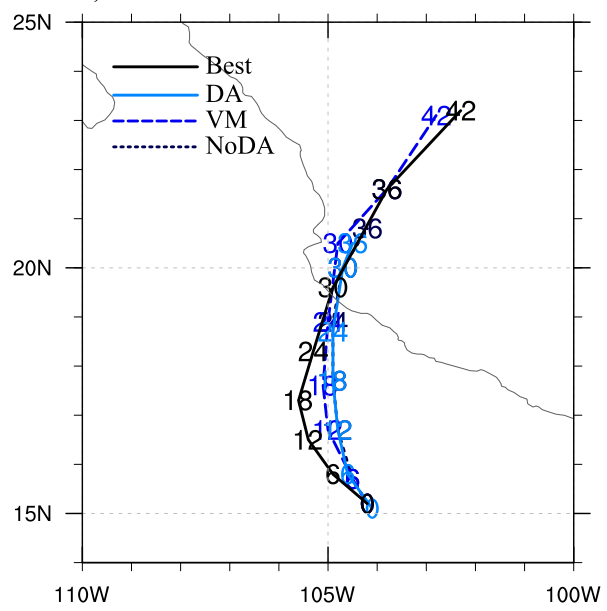

b) MSLP Forecasts

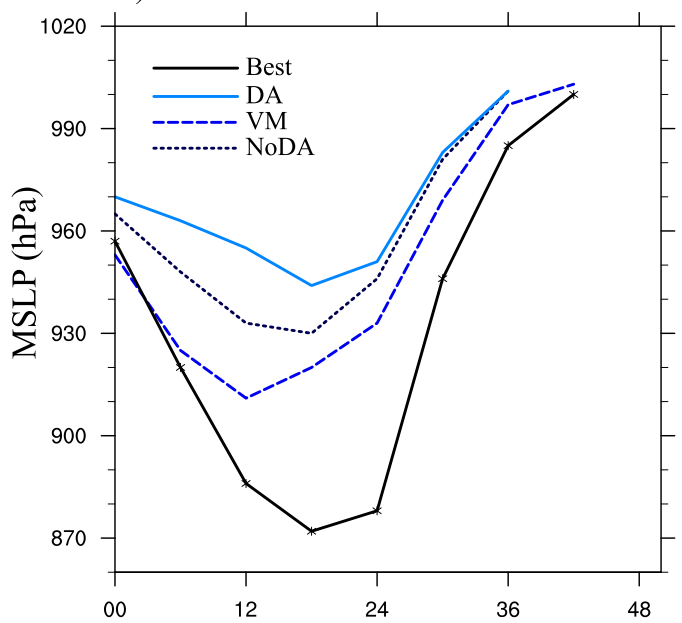

d) Track Error

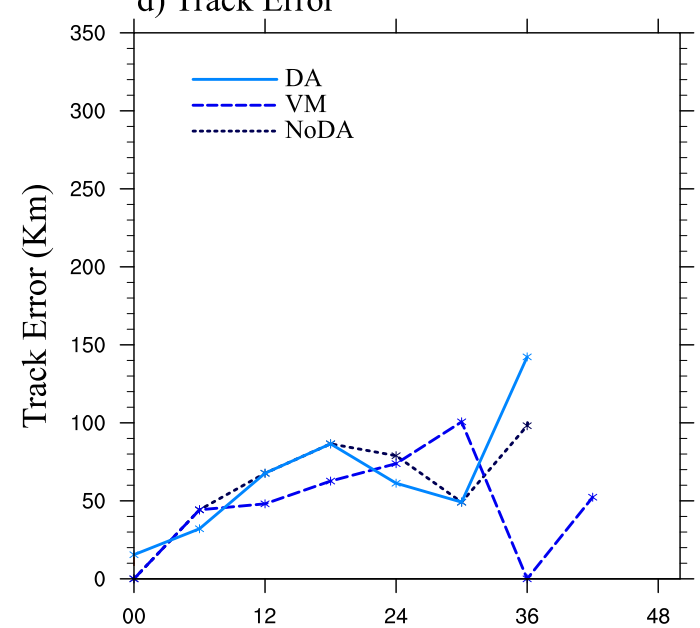

FIG. 5. (a) 10-m Vmax forecast, (b) MSLP forecast, (c) track forecast, and (d) track forecast error for NoDA (dotted navy), VM (dashed blue), DA (solid cyan), and best track (solid black) during Patricia initialized from 1800 UTC 22 Oct 2015. The numbers in (c) indicate the corresponding forecast lead time for each track forecast.

degradation, the track forecast from DA is overall comparable with NoDA (Figs. 5c,d).

\section{b. Why did more persistent spindown occur with a more realistic DA analysis?}

As analyzed in the previous subsection $3 \mathrm{a}, \mathrm{VM}$ creates spuriously strong and large storm with spuriously large supergradient imbalance. However, this analysis produced by VM somehow improves the Vmax and MSLP forecasts upon NoDA. On the contrary, although the DA analysis is more realistic, or more consistent with both theoretical and observed TC structures, the intensity forecast is significantly degraded upon NoDA due to the Vmax spindown. Therefore, diagnostics are performed in this subsection to understand why the more persistent spindown occurs with the more realistic DA analysis but not the unrealistic VM analysis.
The 2-min frequency outputs of the intensity forecasts from different experiments are shown in Fig. 6 to investigate the detailed Vmax and MSLP evolutions during the first two hours. During the time period, NoDA produces a constantly intensifying storm with steady increase in Vmax and steady decrease in MSLP. This steady intensification in NoDA is consistent with the 6-h interval outputs shown in Fig. 5. In comparison with NoDA, although the 6-h interval outputs suggest a slow steady intensification in VM, the higher-frequency outputs show that VM still suffers from a Vmax drop $\left(6 \mathrm{~m} \mathrm{~s}^{-1}\right)$ during the first 10-20 min. Such a Vmax drop is likely to be associated with a dramatic MSLP drop (more than $50 \mathrm{hPa}$ ) in Fig. $6 \mathrm{~b}$. The huge MSLP drop can be attributed to the supergradient imbalance found in Fig. 4, where the pressure gradient is increased by reducing the central pressure to compensate the strong wind field. After a brief (about 

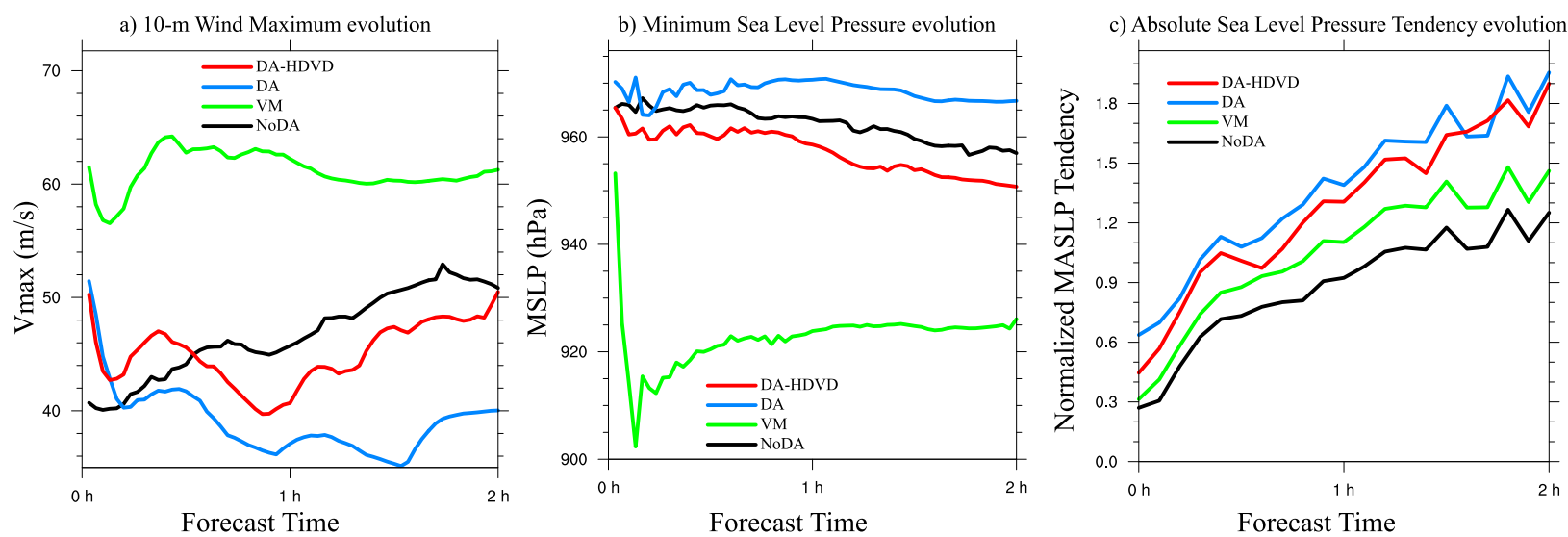

FIG. 6. 2-h evolution of (a) 10-m Vmax, (b) MSLP and (c) NMASPT for NoDA (black), VM (green), DA (cyan) and DA-HDVD (red) initialized from 1800 UTC 22 Oct 2015. The output are plotted every $2 \mathrm{~min}$.

20-25 min) period of decaying and reintensification, the intensity becomes near-stationary. In other words, it takes HWRF a short period of time to regain its initial Vmax value although the VM analyzed vortex is unrealistically supergradient. In comparison to VM, DA does not suffer from the significant MSLP drop due to the more physically balanced initial condition. However, the Vmax in DA drops more (about $16 \mathrm{~m} \mathrm{~s}^{-1}$ ) and longer (about $1 \mathrm{~h}$ ) than VM. DA struggles to intensify and it never regains the initial Vmax strength over the first $6 \mathrm{~h}$. This Vmax drop, struggling to intensify, and failure to recover are reflected as the spindown issue shown in Fig. 5.

To understand why DA and VM evolve differently in the intensity forecast, the temporal evolution of the secondary circulation is investigated and shown in Fig. 7. Since HWRF resets vertical velocity to 0 when it is initialized, ${ }^{2}$ and Vukicevic et al. (2013) suggested that this could be one of the factors that contribute to the spindown issue within their HWRF Ensemble Data Assimilation System (HEDAS) system, an extra experiment NoDA-warm is first conducted to investigate the potential impact of this loss of initial velocity on the secondary circulation evolution (Figs. 7a-e). In NoDA-warm, the initial secondary circulation consists of four major components: a strong inflow in the boundary layer, a strong outflow in the upper level, an updraft in the eyewall connecting the boundary layer inflow and upper-level outflow, and a weak downdraft in the upper-level eye region (Fig. 7a). These inner-core features are typically found in the conceptual model of a mature hurricane (e.g., Liu et al. 1997). Additionally, the inflow and outflow oscillations between the dominant upper-level outflow and boundary layer inflow

\footnotetext{
${ }^{2}$ Restart mode does not function in the HWRF Model when the moving nest configuration is used.
}

are also found in NoDA-warm. As stated in section 1, these vertically oscillating features are consistent with previous studies (Willoughby et al. 1984; Marks and Houze 1987; Stern and Nolan 2011; Stern et al. 2017) and are hypothesized to be related to the supergradient/subgradient unbalanced oscillation around the eyewall (Bryan and Rotunno 2009a). As compared to NoDA-warm, although vertical velocity is set to 0 at the initial time (Fig. 7f), after only $2 \mathrm{~min}$ of model integration, the secondary circulation evolution in NoDA becomes comparable with NoDA-warm (Figs. $7 \mathrm{~b}$ and $7 \mathrm{~g}$ ), suggesting the zeroed out vertical velocity field is not the main cause of the spindown. This high similarity in the secondary circulation evolution between NoDA-warm and NoDA consistently exists over the first 30 min (Figs. 7a-d,f-i), and gradually diverges afterward due to the influences of relocation and GFS replacement in the outmost domain (Figs. 7e,j).

To help visualize the temporal evolution of secondary circulation as analyzed above, a Hovmöller diagram is given in Fig. 8 to mark the time evolution of the eyewall for each experiment. Consistent with Figs. 7a-j, Figs. $8 \mathrm{a}, \mathrm{b}$ again show that although differences can be found, the general feature of the eyewall evolution is overall comparable between NoDA and NoDAwarm, especially during the first $2 \mathrm{~h}$. These results suggest that the resetting of initial vertical velocity in NoDA has minimal impacts on the secondary circulation evolution in the HWRF Model. Nevertheless, the eyewall in both NoDA and NoDA-warm is spuriously large in size $(500-\mathrm{hPa}$ radii about 45 and $40 \mathrm{~km}$, respectively; Figs. 8a,b) compared to the small size (surface RMW about $18 \mathrm{~km}$ ) from the best track. ${ }^{3}$

\footnotetext{
${ }^{3}$ The RMW information is obtained from the postseason b-deck "best tracks" (ftp://ftp.nhc.noaa.gov/atcf/archive/2015/).
} 
a) NoDA-warm @ 00minb) NoDA-warm @,02minc) NoDA-warm @,04min d) NoDA-warm @ 30min e) NoDA-warm @ 60min

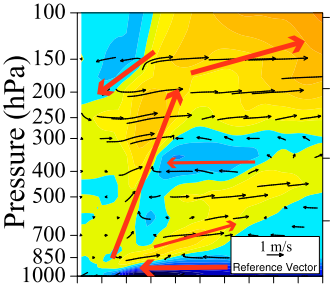

f) NoDA @00 min

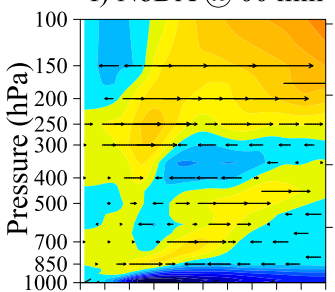

k) VM @ 00 min

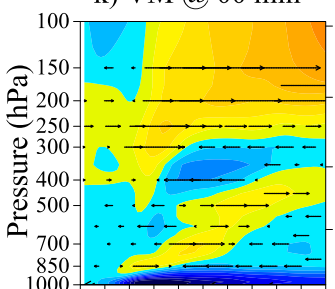

p) DA @ 00 min

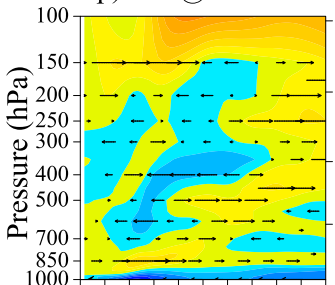

u) DA-HDVD @ 00 min

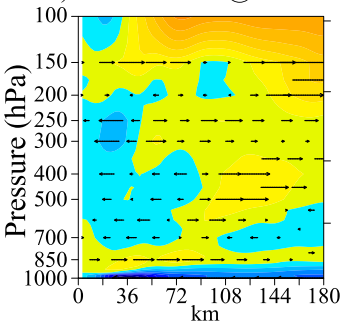

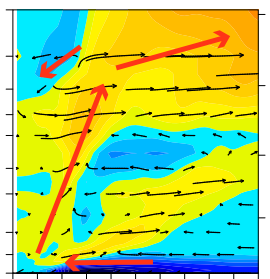

g) NoDA @ 02 min

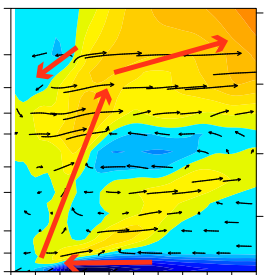

1) VM@02 min

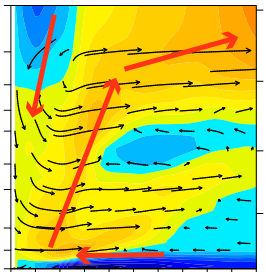

q) DA @ 02 min

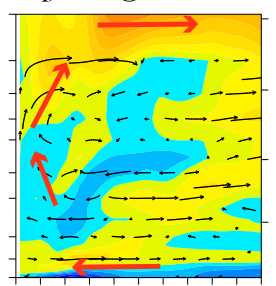

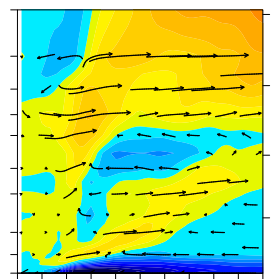

h) NoDA@04 min

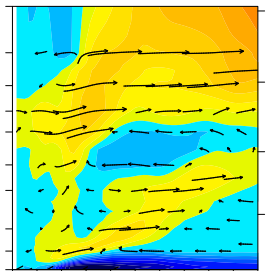

m)VM@04min

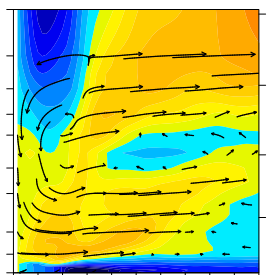

r) DA@04 min

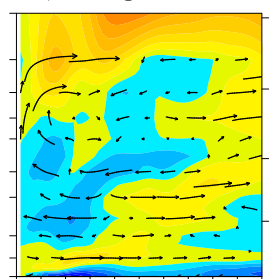

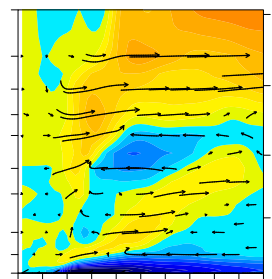

i) NoDA@30 min

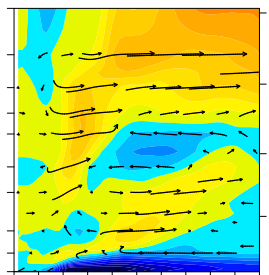

n) VM@30 min

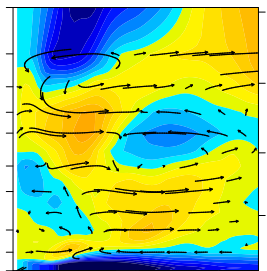

s) DA @30 min

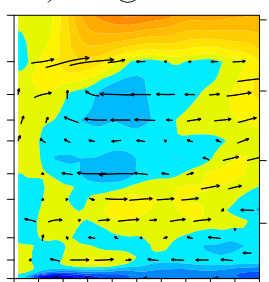

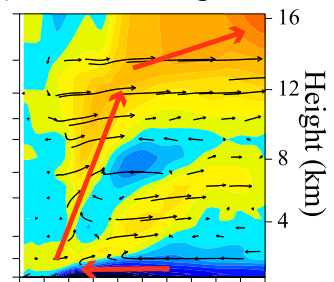

j) NoDA@60 min

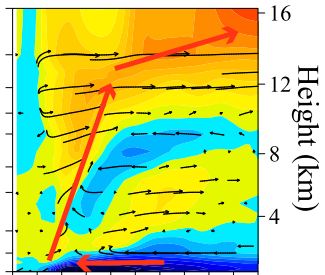

o) VM@60min

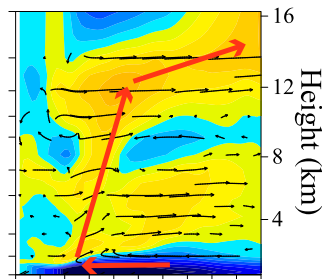

t) DA @ 60 min

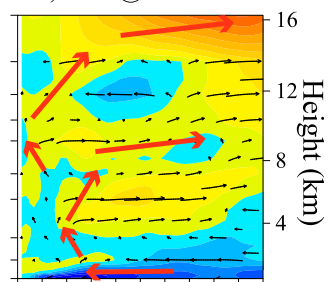

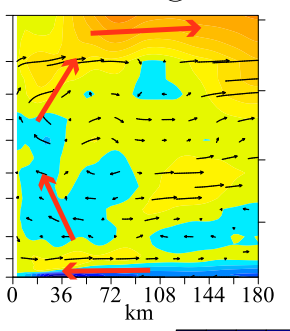
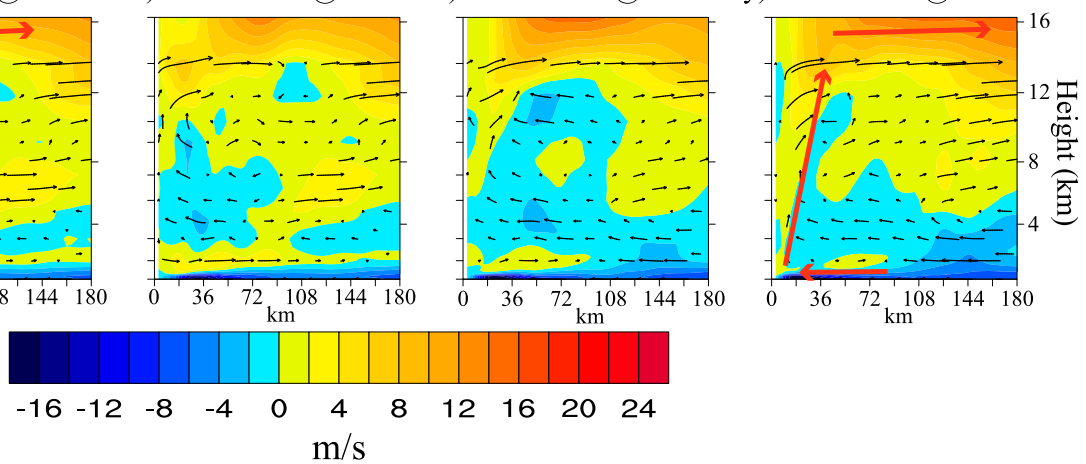

FIG. 7. Time evolution of the azimuthal mean radial wind (shading, $\mathrm{m} \mathrm{s}^{-1}$ ) and secondary circulation (vectors, $\mathrm{m} \mathrm{s}^{-1}$ ) for (a)-(e) NoDAwarm, (f)-(j) NoDA, (k)-(o) VM, (p)-(t) DA, and (u)-(y) DA-HDVD for the (a),(f),(k),(p),(u) initial analysis; (b),(g),(l),(q),(v) 2-min forecast; (c), (h), (m), (r),(w) 4-min forecat; (d),(i),(n),(s),(x) 30-min forecast; and (e),(j),(o),(t),(y) 60-min forecast. Red arrows are added to illustrate the evolution of secondary circulations.

Unlike the similar TC structures between NoDA and NoDA-warm, VM modifies NoDA significantly as shown in Figs. 3 and 4. However, the initial radial wind structures produced by VM are similar to NoDA (Fig. 7k). This result shows that the primary modifications in $\mathrm{VM}$ are through enhancing the tangential wind fields, or the primary circulation. The supergradient imbalance found in VM starts to impact the secondary circulation structures after the model integrates (e.g., 2 min later). For example, spuriously strong downdraft (greater than $6 \mathrm{~m} \mathrm{~s}^{-1}$ ) is 


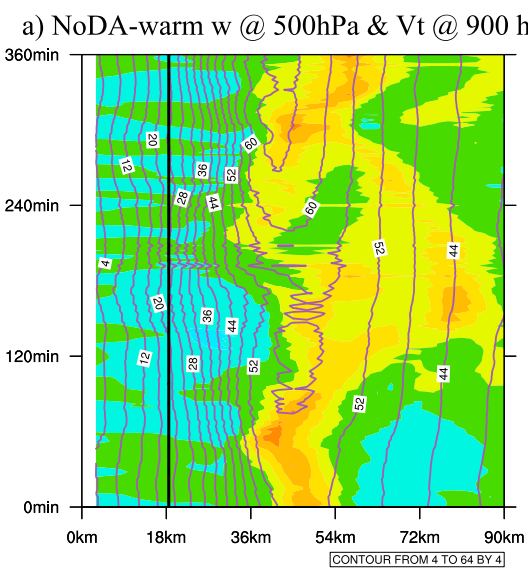

b) NoDAw@500hPa \& Vt@900hPa

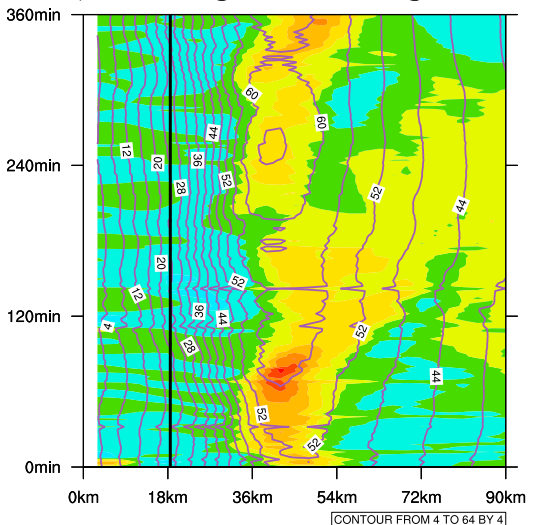

c) VMw @ 500hPa \& Vt @, 900 hPa

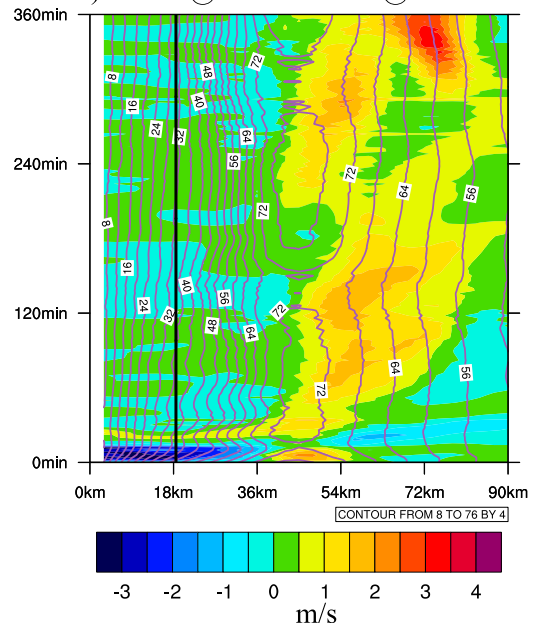

d) DAw@500hPa \& Vt @, $900 \mathrm{hPa}$

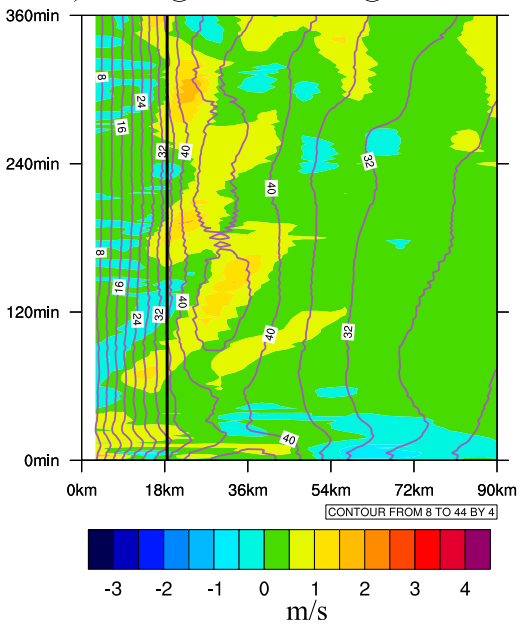

e) DA-HDVDw@500hPa \& Vt @, 900 hPa

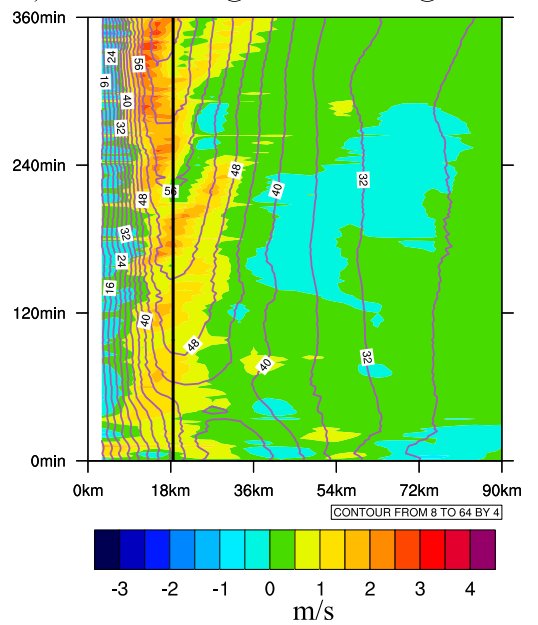

FIG. 8. Radius-time Hovmöller plots of the 500-hPa azimuthal mean vertical velocity (shading, $\mathrm{m} \mathrm{s}^{-1}$ ) and $900-\mathrm{hPa}$ tangential wind (contour, $\mathrm{m} \mathrm{s}^{-1}, 4 \mathrm{~m} \mathrm{~s}^{-1}$ interval) for (a) NoDA-warm, (b) NoDA, (c) VM, (d) DA, and (e) DA-HDVD for the first $6 \mathrm{~h}$. The RMW from the best track is given as a solid black line.

found in the eye region (Figs. 71-n). This strong downdraft is consistent with the dramatic MSLP drop found in Fig. 6. The downdraft warms the eye and hydrostatically lowers the surface pressure as a result of the wind and pressure adjustment. However, regardless of the spuriously strong downdraft in the eye, the typical TC secondary circulation components like the upper-level outflow, low-level inflow and the linking updraft can still be consistently found during the evolution (Figs. $7 \mathrm{k}-\mathrm{O}$ and $8 \mathrm{c}$ ). After one hour of model integration, the overly strong downdraft in the eye region is gradually reduced and the secondary circulation structure is more consistent with NoDA although the upper-level outflow is weaker (Fig. 7o) and the eyewall size is even larger (e.g., 500-hPa radii greater than $40 \mathrm{~km}$; Fig. $8 \mathrm{c}$ ).

According to the TCI dropsonde observations, the outflow of Patricia at the analysis time should be centered around $100 \mathrm{hPa}$ at the outer radii of eyewall (30-60 km; shown in Part II, Fig. 6a). However, the outflow at the outer radii of eyewall is located around $150 \mathrm{hPa}$ in both NoDA and VM, which is lower than observed (Figs. 7f and 7k). DA elevated the outflow to $100 \mathrm{hPa}$ (Fig. 7p), which is more consistent with the observations (shown in Part II). But once the model integrates, it cannot establish a consistent updraft that directly links the elevated upper-level outflow and lowlevel inflow. Instead, the updraft in DA is not only weak below $300 \mathrm{hPa}$, but also oscillated inward and outward in the vertical direction. The upper part of the updraft is also pushed toward the eye such that the upper-eye region is dominated by updraft instead of the typically expected weak downdraft (Fig. 7q). This disorganized secondary circulation is likely because the HWRF Model is not able to support the strong subgradient/ supergradient oscillations in the DA analysis (Fig. 4c) where subgradient produces inflow and supergradient produces outflow near the eyewall. The strong subgradient/supergradient oscillation in the DA analysis 
is consistent with early observational and modeling study of Patricia (Stern et al. 2017). Consequently, the inflow/outflow oscillations within the updraft produce several closed small secondary circulations. This disorganized secondary circulation continues throughout the first hour and is consistent with the Vmax spindown (Fig. 6a). As discussed in section 1, Bryan and Rotunno (2009a) found that the vertical variation associated with the unbalanced flow effects can be damped by stronger radial diffusion. It is hypothesized that one of the reasons that HWRF cannot support the realistically analyzed unbalanced oscillations in the eyewall could be that the horizontal diffusion is too large.

Moreover, even after one hour of model integration, the dominant updraft in the eyewall for DA is still not established (Fig. 7t). Figure $8 \mathrm{~d}$ shows that DA is not able to build up a consistent updraft in the eyewall until about $100 \mathrm{~min}$ later, although the size of eyewall in DA is more consistent with the best track as compared to either NoDA or VM. Furthermore, after one-hour of model integration, the updraft in DA (Fig. 8d) is in general weaker in strength as compared to NoDA (Fig. 8b). This weak updraft cannot reach the realistically elevated upper-level outflow by DA. Therefore, a lower upper-level outflow is therefore spuriously generated during the model integration to satisfy the mass conservation (Fig. 7t). The weak updraft is hypothesized to be related to the unrealistically discontinuous vertical diffusion parameterization as mentioned in section 1 (Fig. 1), where the lack of vertical diffusion at the boundary layer top constrains the upward moisture and energy transport and therefore the updraft triggered by latent heat release in the eyewall is constrained. Such an inefficient vertical energy and moisture transport is reflected by Figs. 9a-o where an unrealistic localized low-level total condensate maximum around $900 \mathrm{hPa}$ is found in all the experiments at any forecast time in HWRF.

Overall, diagnostics in this subsection suggest that the spindown issue in Patricia is a direct response to the secondary circulation evolution. The disorganized and weak secondary circulation is likely to be attributed to the model physics deficiencies such as the unrealistic horizontal and vertical diffusion parameterization configurations. In other words, the spindown happens when the unrealistic model physics parameterization configurations cannot maintain the more realistic analysis produced by DA.

The mean absolute surface pressure tendency (MASPT) is usually used as a measurement of the incompatibility between the initial condition (or analysis) and the numerical model (e.g., Lynch and Huang 1992; Kleist et al. 2009; Wang et al. 2013; Lei and Whitaker, 2016).
Given the surface pressure tendency will likely be dependent on the model physics itself, to facilitate quantifying the "incompatibility" between the model physics and DA analysis, a normalized mean absolute surface pressure tendency (NMASPT) is calculated in Fig. $6 c{ }^{4}$ This NMASPT is calculated by normalizing MASPT with the relatively balanced free forecast in NoDA-Warm and NoDA-HDVD-Warm. The larger the NMASPT, the more incompatible between the model and the DA analysis. Figure $6 \mathrm{c}$ shows that VM only introduces slightly more incompatibility compared to NoDA since the composite vortex added in VM is from historical HWRF Model forecasts and is therefore more model "compatible". In contrast, the confliction between the unrealistic model physics and the realistic analysis by DA shows significantly more incompatibility measured by NMASPT.

Therefore, the spindown issue is hypothesized to be alleviated for the DA experiment when the model physics are improved. The hypothesis will be investigated in the next section.

\section{Can modified model physics alleviate the spindown issue?}

As stated in section $3 b$, improving model physics parameterizations, such as the horizontal and vertical diffusion parameterizations, are hypothesized to help alleviate the spindown issue. Therefore, additional experiments DA-HD and DA-HDVD with physics modifications are conducted and the best performer will be compared with DA in this section to investigate the hypothesis.

\section{a. Impacts of model physics parameterization modifications on TC analyses and intensity forecasts}

Although the 6-h background and ensemble error covariances are different due to the use of different model physics, the DA analyses from these additional experiments are still comparable (Figs. $3 d-f, j-1$ ). The only exception is that DA-HDVD analysis is less symmetric than the DA analysis. This more asymmetry in DA-HDVD is reflected by the Fourier decomposition in the relative vorticity, where DA-HDVD is explained more by the higher-wavenumber components as compared to DA, especially at wavenumber 2 (not shown). However, the more compact wind maximum region in DA-HDVD seems to be more consistent with the HRD

\footnotetext{
${ }^{4}$ Sensitivity experiments suggest that deviates of NoDA from one are primarily due to the replacement of GFS analysis in the outermost domain and the VR process (not shown).
} 
a) NoDA@00 min

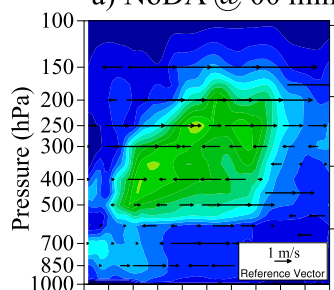

f) VM @, $00 \mathrm{~min}$

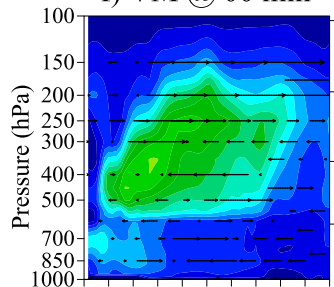

k) DA@00 min

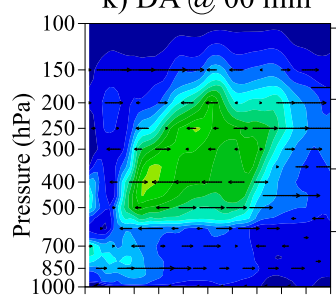

p) DA-HDVD @ 00 minq

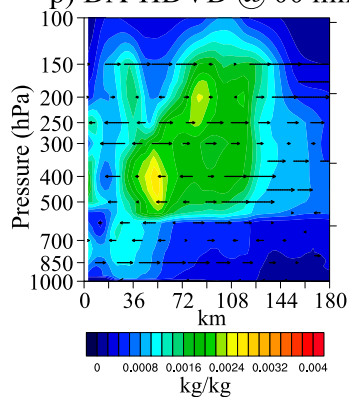

b) NoDA @ 02 min

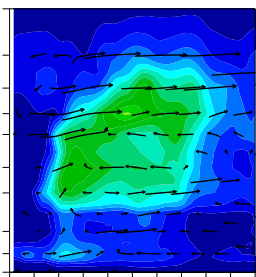

g) VM@02 min

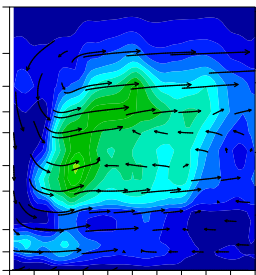

1) DA@02 min

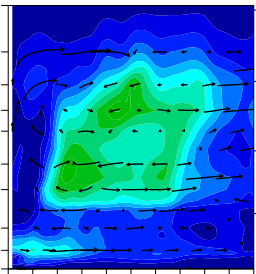

c) NoDA@04 min

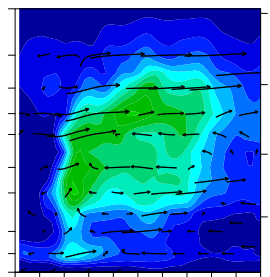

h) VM@ 04 min

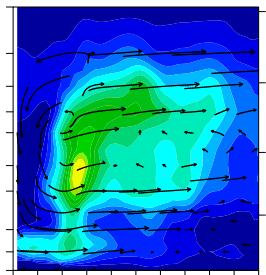

m) DA@04 min

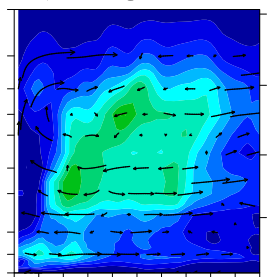

d) NoDA@30 min

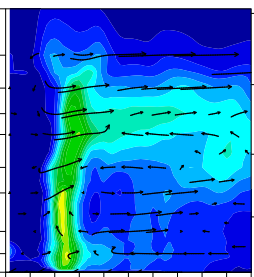

i) VM@ $30 \mathrm{~min}$

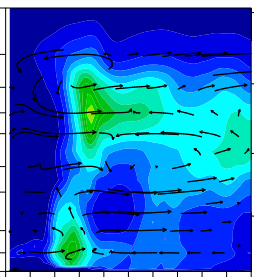

n) DA @30 min

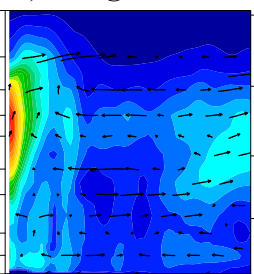

e) NoDA @ 60 min

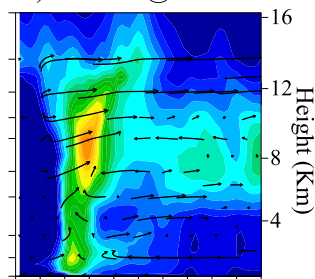

j) VM@60 min

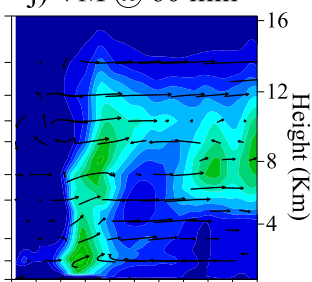

o) DA@60 min

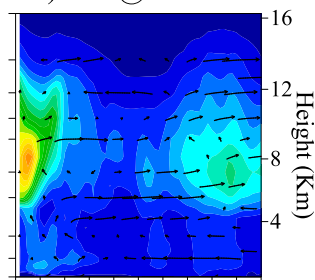

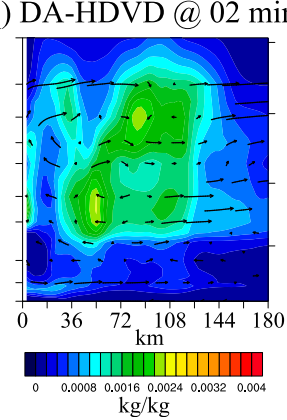
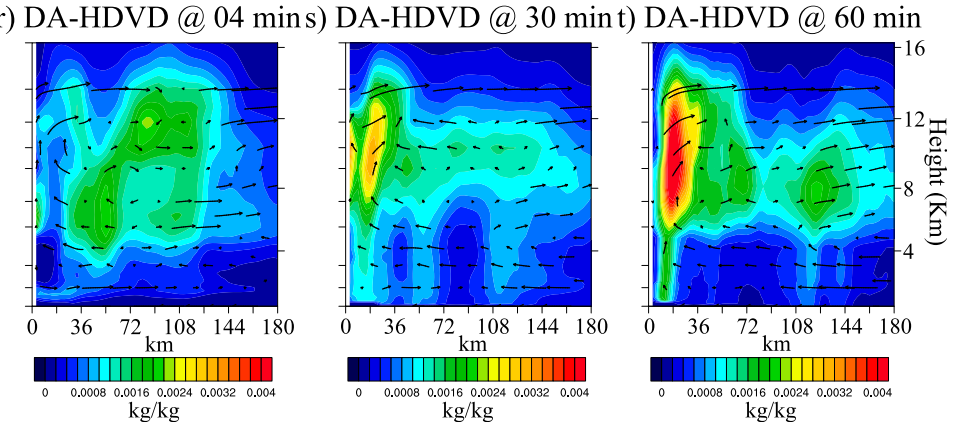

FIG. 9. As in Fig. 7, but for the shading for azimuthal mean total condensate $\left(\mathrm{kg} \mathrm{kg}^{-1}\right)$ for (a)-(e) NoDA, (f)-(j) VM, (k)-(o) DA, and (p)-(t) DA-HDVD for the (a),(f),(k),(p) initial analysis; (b),(g),(l),(q) 2-min forecast; (c),(h),(m),(r) 4-min forecat; (d),(i),(n),(s) 30-min forecast; and $(\mathrm{e}),(\mathrm{j}),(\mathrm{o}),(\mathrm{t}) 60$-min forecast.

radar composite. Overall, all the experiments using the hybrid 3DEnVar with inner-core observations can capture the major TC features at different levels consistent with the verifications as discussed in section 3a. For example, the contracted storm size and the weaker wind speed to the southwest of Patricia are both captured.

Nevertheless, the intensity and track forecasts initialized from these similar analyses are different as shown in Fig. 10. Using a reduced horizontal diffusion, DA-HD improves the MSLP forecast and shows apparent alleviation of spindown in the Vmax forecast as compared to DA (Figs. 10a,b) although no improvement is found in the track forecast (Figs. 10c,d). Without the significant spindown issue, the simulated peak intensity of DA-HD is increased over the peak intensity of DA, and is closer to the best track. This improved Vmax forecast suggests that the overly large horizontal diffusion is likely one of the reasons for the spindown issue during the prediction of Patricia as hypothesized. But the RI rate in DA-HD is still slower than the best track and so is the peak intensity. This slow and weak intensification in DA-HD is likely still due to the lack of strong updraft connecting the realistically elevated upper-level outflow as suggested in section $3 \mathrm{~b}$ (not shown).

Using the modified turbulent mixing parameterization scheme on top of the reduced horizontal diffusion in DAHDVD shows further improvement in Vmax, MSLP and track forecasts upon DA-HD (Figs. 10a-d). The first 6-h RI trend in DA-HDVD is now comparable with the best track and the spindown is significantly alleviated. Specially, the peak Vmax and MSLP values in DA-HDVD is now $20 \mathrm{~m} \mathrm{~s}^{-1}$ larger and $44 \mathrm{hPa}$ smaller than DA, respectively, 
a) Vmax Forecasts

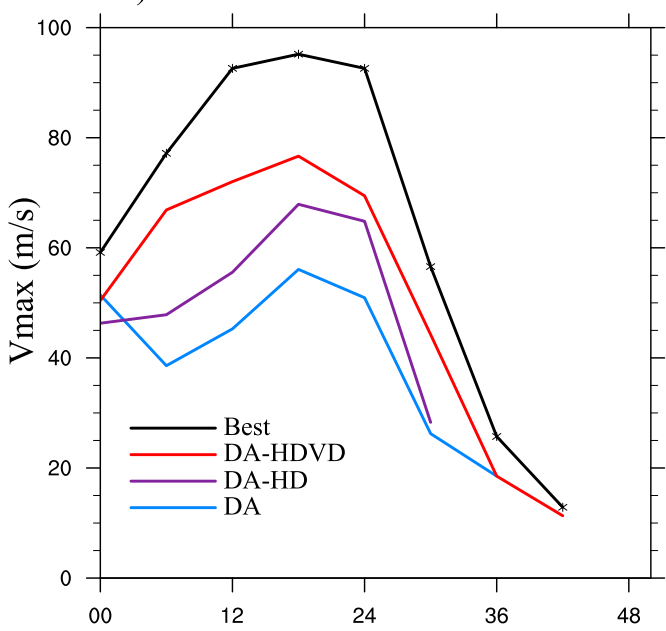

c) Track Forecasts

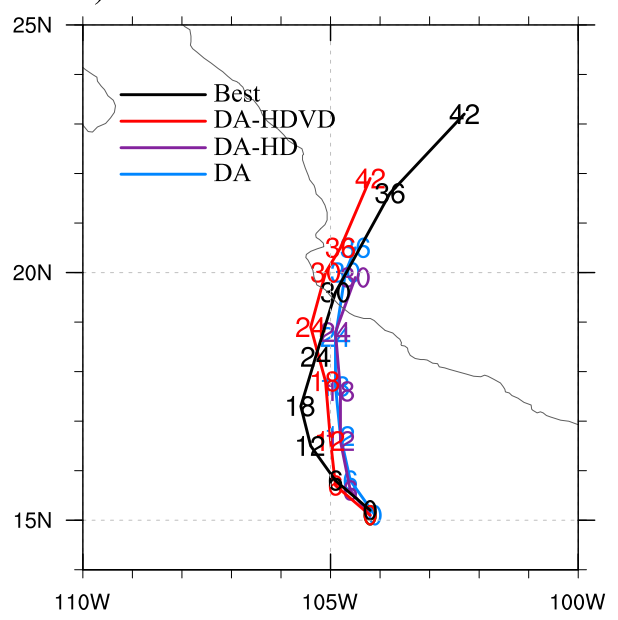

b) MSLP Forecasts

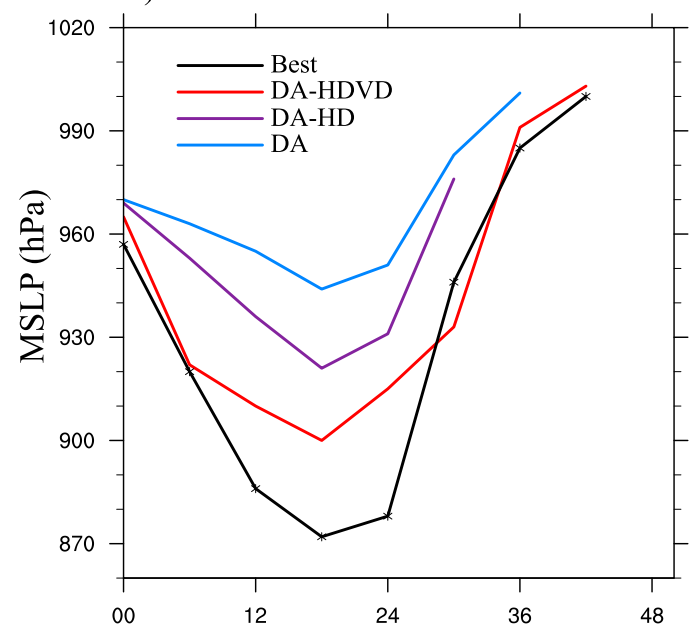

d) Track Error

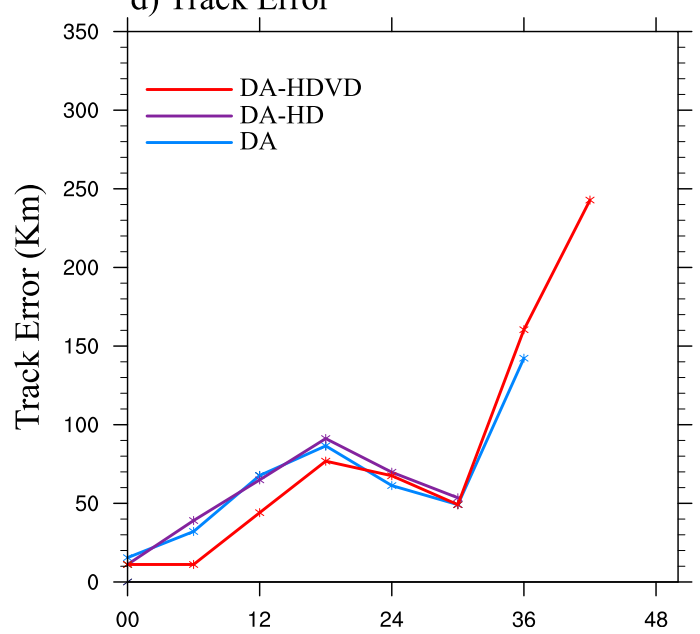

FIG. 10. As in Fig. 5, but for DA (cyan), DA-HD (purple), DA-HDVD (red), and best track (black).

more consistent with the best track. More detailed investigations on how the spindown is alleviated with DAHDVD will be presented in the next subsection $4 \mathrm{~b}$.

In general, this subsection confirms the hypothesis proposed at the end of section 3b: improved model physics are needed to support and maintain the realistically analyzed TC structure from DA during the forecast. For the Patricia forecast in particular, both the modified turbulent mixing parameterization scheme and the reduced horizontal diffusion are important for improving the intensity forecast initialized from an improved analysis.

\section{b. How can the modified model physics parameterizations alleviate spindown?}

To further understand how the improved model physics can alleviate the spindown issue, detailed diagnostics similar to section $3 b$ are performed in this subsection for the best performing DA-HDVD experiment.
First of all, the frequent output of Vmax and MSLP in Figs. $6 \mathrm{a}$ and $6 \mathrm{~b}$ shows that although alleviated and is not found in the 6-h frequency output, DA-HDVD still suffers from a Vmax drop of about $12 \mathrm{~m} \mathrm{~s}^{-1}$ within the first hour. However, this Vmax drop in DA-HDVD is $25 \%$ smaller than DA, and DA-HDVD intensifies rapidly and consistently after the first hour of adjustment. The RI in DA-HDVD after hour 1 compensates the Vmax drop and restores the Vmax to the initial value around hour 2. The Vmax in DA-HDVD keeps growing afterward. Consistent with the Vmax evolution, the MSLP evolution in DA-HDVD shows smaller magnitude of adjustments as compared with DA in the first hour and is followed by a steady intensification afterward.

The secondary circulation evolution of DA-HDVD (Figs. $7 \mathrm{u}-\mathrm{y}$ ) shows that the vertical sub/supergradient oscillation in the eyewall is still not well supported during the first several minutes of model integration. 
However, the issue is less significant and less persistent due to the reduced horizontal diffusion. At the end of hour one, there is a clear outward updraft that connects the low-level inflow and realistically elevated upperlevel outflow (Fig. 7y). Consistently, Fig. 8e shows that the eyewall can be built up quickly in DA-HDVD and well maintained during the evolution. The $500-\mathrm{hPa}$ radius of eyewall in DA-HDVD is comparable with or even slightly smaller than the RMW given by the best track. The enhanced updraft is likely to be a result of applying the modified turbulent mixing parameterization scheme. Elimination of the vertical diffusion discontinuity in the new parameterization scheme (Fig. 1) facilitates the vertical communication of heat and moisture. Consequently, no localized low-level total condensate maximum is found in Figs. 9p-t, which suggests that the hydrometeors are well mixed in the eyewall region and can release more latent heat in the eyewall (Fig. 9t), and eventually enhanced the secondary circulation. As discussed in section $3 \mathrm{~b}$, the NMASPT is calculated for DA-HDVD as well. Figure $6 \mathrm{c}$ shows that consistent with the spindown alleviation, DA-HDVD reduces the model-analysis incompatibility as compared to DA.

Overall, comparisons from Fig. 6 to Fig. 9 suggest that the spindown issue in Patricia is likely to be a consequence of the conflict between the unrealistic model physics parameterization and the more realistic DA analysis as hypothesized in section $3 \mathrm{~b}$. Reducing the confliction by either using an unrealistic but model generated analysis as VM or using a more realistically improved model physics parameterization can help alleviate the spindown issue. However, there is still more incompatibility in DA-HDVD in comparison with VM (Fig. 6c) and DA-HDVD do still suffer from the short-term Vmax drop. These results suggest that further tunings and investigations in the model physics and further improvement of the DA are needed in the future.

\section{How does model resolution impact on the TC intensity forecast?}

As shown in section 4, the improved model physics parameterization can help alleviate the spindown issue and improve the TC intensity forecast significantly. However, the forecasted maximum peak intensity is still about $20 \mathrm{~m} \mathrm{~s}^{-1}$ or $30 \mathrm{hPa}$ weaker than the peak Vmax or MSLP recorded by the best track. This remaining gap between the two peak intensities is likely due to the insufficient model resolution considering the small size of Patricia as stated in section 1 . Therefore, an experiment has been conducted in this study to investigate the impact of model resolution on the peak intensity as well as the spindown issue.
Similar to Fig. 5, the Vmax, MSLP and track forecasts of the model resolution experiments are verified against the best track in Fig. 11. Without changing the model physics parameterization configuration, DA-Hi shows slightly alleviated Vmax spindown and a significant improvement in the peak intensity in comparison with DA. However, this spindown alleviation through the resolution increase is not as significant as the model physics parameterization improvement. This result suggests the spindown is largely attributed to the model physics errors rather than the relatively coarse model resolution. While the peak intensity is improved in DA-Hi as compared to DA, the track forecast is somehow degraded because the predicted TC moves too fast. Specifically, DA-Hi makes landfall after hour 18 while the best track shows landfall close to hour 30 .

Since the spindown is already alleviated in DA-HDVD, increasing model resolution with the improved model physics in DA-HDVDHi shows significantly more improvement in the intensification rate and peak intensity. Although the timing is shifted $6 \mathrm{~h}$ earlier, the peak Vmax in DA-HDVDHi is now comparable with the best track. The early peak intensity in DA-HDVDHi is also due to the overfast prediction of track similar to DA-Hi (Figs. 11c,d).

Altogether, Fig. 11 suggests that the model resolution can be an important factor for maximum peak intensity prediction for HWRF Model when the model physics are properly modified, but the model resolution is not as important as the model physics in the spindown alleviation for this case.

\section{Summary and discussion}

A newly developed GSI-based, continuously cycled, dual-resolution hybrid EnKF-Var DA system for HWRF (Lu et al. 2017b) is upgraded to be consistent with the 2015 operational HWRF at a higher model resolution. Using this upgraded system, abundant field campaign inner-core and near inner-core observations together with the enhanced CIMSS AMVs collected during Hurricane Patricia (2015) are assimilated to provide a realistic 3D analysis of the storm. As Part I of the two-part study, this paper aims at investigating the source of the spindown issue associated with the more realistic DA analysis. Diagnostics have shown that the analysis is consistent with various observations. Additionally, the analysis produced by DA is more consistent with the GWB and unbalanced force theories. In contrast, VM creates a spuriously strong supergradient imbalance throughout the inner-core to near inner-core region. However, the realistic analysis produced by DA is found to experience significant and persistent Vmax spindown (e.g., Vmax drop lasts longer than $6 \mathrm{~h}$ ) which jeopardizes the subsequent intensity forecast. On the other 
a) Vmax Forecasts

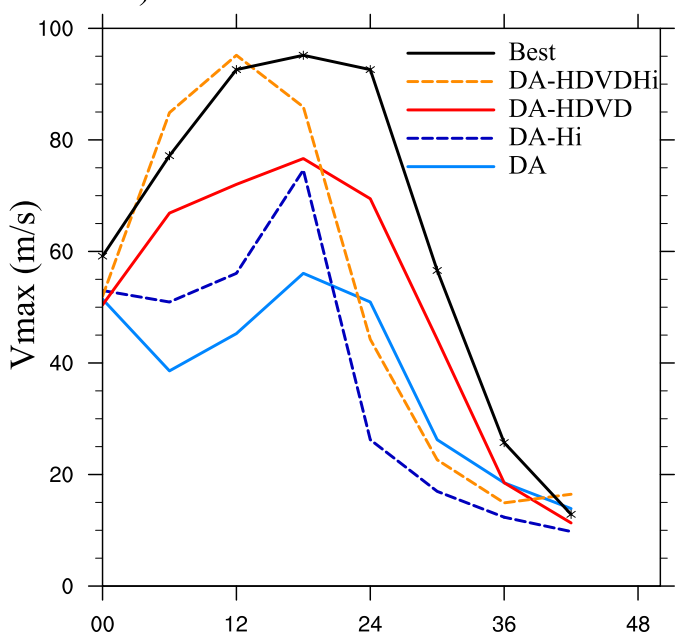

c) Track Forecasts

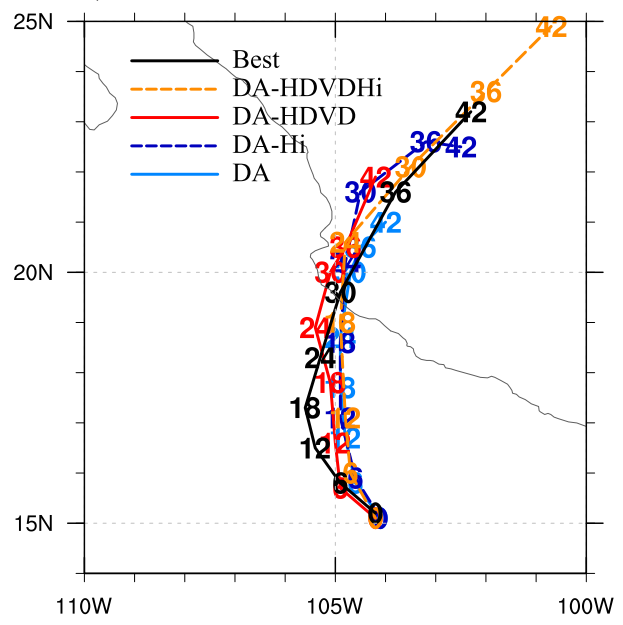

b) MSLP Forecasts

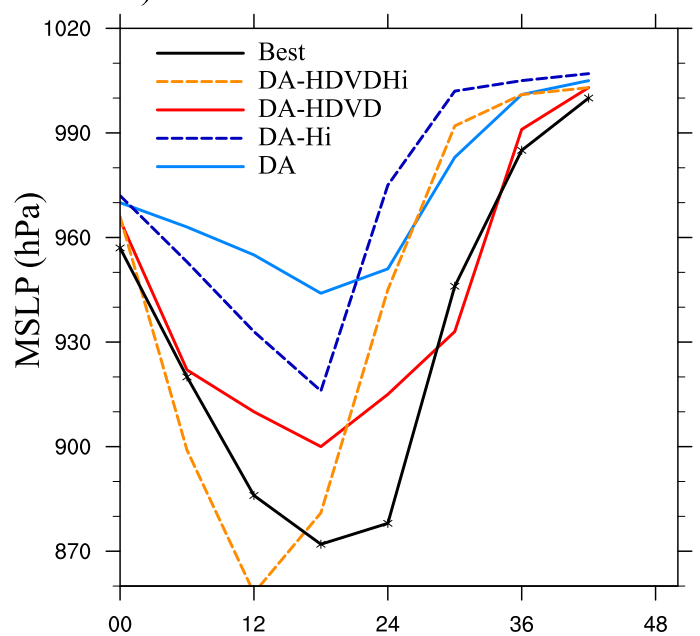

d) Track Error

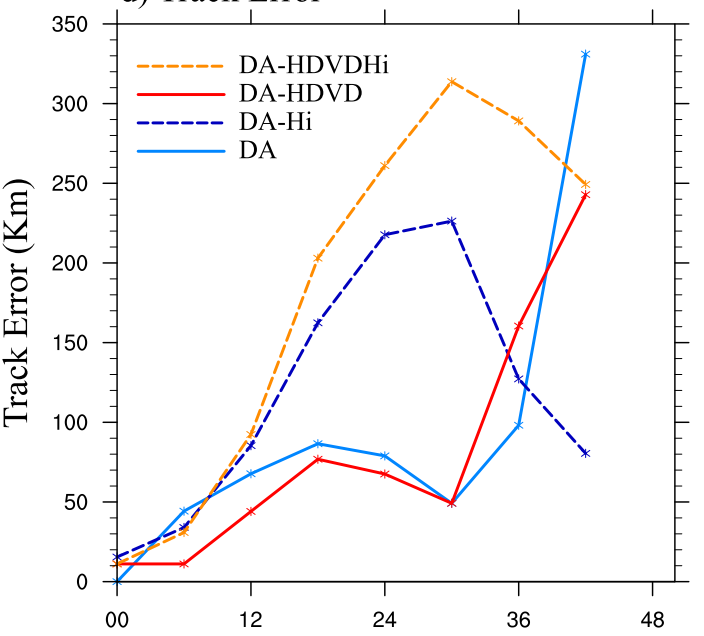

FIG. 11. As in Fig. 5, but for DA (solid cyan), DA-Hi (dashed blue), DA-HDVD (solid red), DA-HDVDHi (dashed orange), and best track (solid black).

hand, the spurious analysis produced through VM is found to produce better intensity forecast without apparent long-lasting Vmax drop. Therefore, diagnostics are performed in this study to understand why the more persistent spindown occur when initialized with the more realistic DA analysis but not with the spurious VM analysis.

Frequent outputs from HWRF Model show that Vmax drop happens in both DA and VM. The Vmax drop in VM is attributed to the significant wind and pressure adjustment caused by the supergradient imbalance at the initial time. This model adjustment in VM is significant but brief. Vmax is recovered to the strength of the analysis quickly and slowly intensifies afterward. However, the Vmax drop in DA is more severe and persistent than the Vmax drop in VM. The evolution of secondary circulation and eyewall indicates that this significant Vmax drop in
DA is attributed to the defective model physics parameterization schemes that cannot maintain the realistic sub/ supergradient oscillations associated inflow/outflow oscillations around the eyewall. Additionally, the realistically elevated upper-level outflow by DA is also not supported by the model due to the unrealistically weak secondary circulation even after the model resumes its balance. It is hypothesized from these results that better HWRF Model physics such as the turbulent horizontal and vertical diffusion parameterizations are needed to alleviate the spindown initialized by the analysis produced by DA.

Further diagnostics with modified model turbulent diffusion parameterizations are therefore conducted in this study to investigate the hypothesis. Results show that using a reduced horizontal diffusion parameterization configuration is able to better maintain the sub/supergradient oscillation from the DA analysis and therefore significantly 
alleviated the spindown issue. Further applying the modified turbulent mixing parameterization scheme with improved vertical diffusion profile significantly increased the intensification rate and peak intensity with enhanced secondary circulation. However, it should be noted that this study is not trying to suggest that the reduction in the horizontal diffusion or the change of vertical diffusion profile is a final resort to resolve the spindown issue. Rather, the experiments conducted in this study demonstrate that the model physics do play an important role in the spindown alleviation and a realistic DA analysis requires a compatible, realistic model physics to improve the TC intensity forecast.

Considering the small size of Patricia, some initial experiments are also conducted in this study to investigate the impacts of increased model resolution in the intensity forecast. Results show that by increasing the model resolution, the spindown issue can be alleviated and the intensification rate and therefore peak intensity can be increased significantly. However, the spindown alleviation from the resolution increase is not as significant as the alleviation from the model physics modification. These results suggest that the model resolution is one key factor that limits the intensification rate and peak intensity, but the model physics plays a more important role in the spindown alleviation in this case. Additionally, the predicted TC moves overly fast with the finer-resolution experiments, which requires further investigations in the future work. In Part II, the impact of various field campaign and enhanced CIMSS AMV observations on the analysis and subsequent forecast of Patricia using the improved physics are discussed. This study focuses on the Vmax spindown with a time scale of $6 \mathrm{~h}$ or more, based on definition of spindown from early studies (Bernardet et al. 2015; Zhou et al. 2015a,b; Tong et al. 2018). The Vmax drop at a much shorter time scale (e.g., over the first 10-20 min shown in Fig. 6a) is worth additional investigation in future studies.

Acknowledgments. The research documented in this paper is supported by NOAA Grants NA14NWS4680021 and NA16NWS4680028 and the ONR Grants N00014-141-0125 and N000141712111. The experiments are performed on the NOAA supercomputer jet. Some results and descriptions were included in the abstract of the authors' AMS conference presentation, the abstract of the first author's dissertation seminar, and progress reports to the funding agencies. We are grateful to Ping Zhu, Jun Zhang, Jason Sippel, Avichal Mehra, and Vijay Tallapragada for helpful discussions.

\section{REFERENCES}

Aksoy, A., S. D. Aberson, T. Vukicevic, K. J. Sellwood, S. Lorsolo, and X. J. Zhang, 2013: Assimilation of high-resolution tropical cyclone observations with an ensemble Kalman filter using NOAA/AOML/HRD's HEDAS: Evaluation of the 2008-11 vortex-scale analyses. Mon. Wea. Rev., 141, 1842-1865, https:// doi.org/10.1175/MWR-D-12-00194.1.

Barker, D. M., W. Huang, Y.-R. Guo, A. J. Bourgeois, and Q. N. Xiao, 2004: A three-dimensional variational data assimilation system for MM5: Implementation and initial results. Mon. Wea. Rev., 132, 897-914, https://doi.org/10.1175/1520-0493(2004) 132<0897:ATVDAS > 2.0.CO;2.

Bauer, P., A. J. Geer, P. Lopez, and D. Salmond, 2010: Direct 4D-Var assimilation of all-sky radiances. Part I: Implementation. Quart. J. Roy. Meteor. Soc., 136, 1868-1885, https://doi.org/ 10.1002/qj.659.

_ similation at operational NWP centres. Quart. J. Roy. Meteor. Soc., 137, 1934-1951, https://doi.org/10.1002/qj.905.

Bernardet, L., V. Tallapragada, C. Holt, S. Trahan, M. Biswas, L. Carson, H. Shao, and C. Zhou, 2015: Transition of research to the operational hurricane WRF Model: The role of the Developmental Testbed Center. Sixth NOAA Testbeds and Proving Grounds Workshop, Boulder, CO, NOAA, https:// www.testbeds.noaa.gov/events/2015/workshop/presentations/ wed-bernardet-hwrf.pdf.

Braun, S. A., M. T. Montgomery, and Z. Pu, 2006: High-resolution simulation of Hurricane Bonnie (1998). Part I: The organization of eyewall vertical motion. J. Atmos. Sci., 63, 19-42, https://doi.org/10.1175/JAS3598.1.

Bryan, G. H., 2012: Effects of surface exchange coefficients and turbulence length scales on the intensity and structure of numerically simulated hurricanes. Mon. Wea. Rev., 140, 11251143, https://doi.org/10.1175/MWR-D-11-00231.1.

_ , and R. Rotunno, 2009a: Evaluation of an analytical model for the maximum intensity of tropical cyclones. J. Atmos. Sci., 66, 3042-3060, https://doi.org/10.1175/2009JAS3038.1.

$\longrightarrow$, and $-2009 \mathrm{~b}$ : The maximum intensity of tropical cyclones in axisymmetric numerical model simulations. Mon. Wea. Rev., 137, 1770-1789, https://doi.org/10.1175/2008MWR2709.1.

Chen, H., and D.-L. Zhang, 2013: On the rapid intensification of Hurricane Wilma (2005). Part II: Convective bursts and the upper-level warm core. J. Atmos. Sci., 70, 146-162, https://doi.org/ 10.1175/JAS-D-12-062.1.

Cram, T. A., J. Persing, M. T. Montgomery, and S. A. Braun, 2007: A Lagrangian trajectory view on transport and mixing processes between the eye, eyewall, and environment using a high-resolution simulation of Hurricane Bonnie (1998). J. Atmos. Sci., 64, 1835-1856, https://doi.org/10.1175/ JAS3921.1.

Doyle, J. D., and Coauthors, 2017: A view of tropical cyclones from above: The Tropical Cyclone Intensity (TCI) Experiment. Bull. Amer. Meteor. Soc., 98, 2113-2134, https://doi.org/10.1175/ BAMS-D-16-0055.1.

Emanuel, K. A., 1986: An air-sea interaction theory for tropical cyclones. Part I: Steady-state maintenance. J. Atmos. Sci. 43, 585-605, https://doi.org/10.1175/1520-0469(1986)043<0585: AASITF $>2.0 . \mathrm{CO} ; 2$.

, 1995: Sensitivity of tropical cyclones to surface exchange coefficients and a revised steady-state model incorporating eye dynamics. J. Atmos. Sci., 52, 3969-3976, https://doi.org/ 10.1175/1520-0469(1995)052<3969:SOTCTS > 2.0.CO;2. 
— 1997: Some aspects of hurricane inner-core dynamics and energetics. J. Atmos. Sci., 54, 1014-1026, https://doi.org/ 10.1175/1520-0469(1997)054<1014:SAOHIC >2.0.CO;2.

Fierro, A. O., and J. M. Reisner, 2011: High-resolution simulation of the electrification and lightning of Hurricane Rita during the period of rapid intensification. J. Atmos. Sci., 68, 477-494, https://doi.org/10.1175/2010JAS3659.1.

Gamache, J., 2005: Real-time dissemination of hurricane wind fields determined from airborne Doppler radar data realtime dissemination of hurricane wind fields determined. JHT Project Final Rep., 38 pp., http://www.nhc.noaa.gov/ jht/2003-2005reports/DOPLRgamache_JHTfinalreport.pdf.

Geer, A. J., and Coauthors, 2018: All-sky satellite data assimilation at operational weather forecasting centres. Quart. J. Roy. Meteor. Soc., 144, 1191-1217, https://doi.org/10.1002/qj.3202.

Gopalakrishnan, S. G., F. Marks, J. A. Zhang, X. Zhang, J.-W. Bao, and V. Tallapragada, 2013: A study of the impacts of vertical diffusion on the structure and intensity of the tropical cyclones using the high-resolution HWRF system. J. Atmos. Sci., 70, 524-541, https://doi.org/10.1175/JAS-D-11-0340.1.

Guimond, S. R., G. M. Heymsfield, P. D. Reasor, and A. C. Didlake, 2016: The rapid intensification of Hurricane Karl (2010): New remote sensing observations of convective bursts from the Global Hawk platform. J. Atmos. Sci., 73, 3617-3639, https://doi.org/ 10.1175/JAS-D-16-0026.1.

HRD, 2015: Hurricane Research Division dataset during Hurricane Patricia. NOAA, accessed 19 November 2017, http://www.aoml. noaa.gov/hrd/Storm_pages/patricia2015/.

Janjić, Z. I., 1990: The step-mountain coordinate: Physical package. Mon. Wea. Rev., 118, 1429-1443, https://doi.org/ 10.1175/1520-0493(1990)118<1429:TSMCPP > 2.0.CO;2.

Kimberlain, T. B., E. S. Blake, and J. P. Cangialosi, 2016: National Hurricane Center tropical cyclone report: Hurricane Patricia (20-24 October 2015). NOAA/NHC Rep. EP202015, 32 pp., https://www.nhc.noaa.gov/data/tcr/EP202015_Patricia.pdf.

Kleist, D. T., D. F. Parrish, J. C. Derber, R. Treadon, R. M. Errico, and R. Yang, 2009: Improving incremental balance in the GSI 3DVAR analysis system. Mon. Wea. Rev., 137, 1046-1060, https://doi.org/10.1175/2008MWR2623.1.

Kurihara, Y., M. A. Bender, R. E. Tuleya, and R. J. Ross, 1995: Improvements in the GFDL Hurricane Prediction System. Mon. Wea. Rev., 123, 2791-2801, https://doi.org/10.1175/ 1520-0493(1995)123<2791:IITGHP>2.0.CO;2.

_ , R. E. Tuleya, and M. A. Bender, 1998: The GFDL hurricane prediction system and its performance in the 1995 hurricane season. Mon. Wea. Rev., 126, 1306-1322, https://doi.org/ 10.1175/1520-0493(1998)126<1306:TGHPSA > 2.0.CO;2.

Lei, L., and J. S. Whitaker, 2016: A four-dimensional incremental analysis update for the ensemble Kalman filter. Mon. Wea. Rev., 144, 2605-2621, https://doi.org/10.1175/MWR-D-15-0246.1.

Li, Y., X. Wang, and M. Xue, 2012: Assimilation of radar radial velocity data with the WRF hybrid ensemble-3DVAR system for the prediction of Hurricane Ike (2008). Mon. Wea. Rev., 140, 3507-3524, https://doi.org/10.1175/MWR-D-12-00043.1.

Liu, Q., T. Marchok, H. Pan, M. Bender, and S. Lord, 2000: Improvements in hurricane initialization and forecasting at NCEP with global and regional (GFDL) models. NCEP Office Note 472, 7 pp., http://citeseerx.ist.psu.edu/viewdoc/download; jsessionid $=81596$ D2DF1D48C9246DBE3F5E0FE2D4B? doi $=10.1 \cdot 1.516 .4663 \&$ rep $=$ rep1\&type $=$ pdf.

- S. Lord, N. Surgi, Y. Zhu, R. Wobus, Z. Toth, and T. Marchok, 2006: Hurricane relocation in Global Ensemble Forecast System. 27th Conf.on Hurricanes and Tropical
Meteorology, Monterey, CA, Amer. Meteor. Soc., P5.13, https://ams.confex.com/ams/pdfpapers/108503.pdf.

Liu, Y., D.-L. Zhang, and M. K. Yau, 1997: A multiscale numerical study of Hurricane Andrew (1992). Part I: Explicit simulation and verification. Mon. Wea. Rev., 125, 3073-3093, https://doi.org/10.1175/1520-0493(1997)125<3073: $\mathrm{AMNSOH}>2.0 . \mathrm{CO} ; 2$.

Lu, X., X. Wang, Y. Li, M. Tong, and X. Ma, 2017a: GSI-based ensemble-variational hybrid data assimilation for HWRF for hurricane initialization and prediction: Impact of various error covariances for airborne radar observation assimilation. Quart. J. Roy. Meteor. Soc., 143, 223-239, https://doi.org/ 10.1002/qj.2914.

$\longrightarrow,-$, M. Tong, and V. Tallapragada, 2017b: GSI-based, continuously cycled, dual-resolution hybrid ensemble-variational data assimilation system for HWRF: System description and experiments with Edouard (2014). Mon. Wea. Rev., 145, 48774898, https://doi.org/10.1175/MWR-D-17-0068.1.

Lynch, P., and X. Huang, 1992: Initialization of the HIRLAM model using a digital filter. Mon. Wea. Rev., 120,1019-1034, https://doi.org/ 10.1175/1520-0493(1992)120<1019:IOTHMU > 2.0.CO;2.

Marks, F. D., and R. A. Houze, 1987: Inner core structure of Hurricane Alicia from airborne Doppler radar observations. J. Atmos. Sci., 44, 1296-1317, https://doi.org/10.1175/1520-0469(1987)044<1296: ICSOHA $>2.0 . \mathrm{CO} ; 2$.

Montgomery, M. T., and R. K. Smith, 2014: Paradigms for tropicalcyclone intensification. Aust. Meteor. Ocean J., 64, 37-66, https://doi.org/10.22499/2.6401.005.

— , V. A. Vladimirov, and P. V. Denissenko, 2002: An experimental study on hurricane mesovortices. J. Fluid Mech., 471, 1-32, https://doi.org/10.1017/S0022112002001647.

_, M. E. Nicholls, T. A. Cram, and B. Saunders, 2006: A vortical hot tower route to tropical cyclogenesis. J. Atmos. Sci., 63, 355-386, https://doi.org/10.1175/JAS3604.1.

Nguyen, M. C., M. J. Reeder, N. E. Davidson, R. K. Smith, and M. T. Montgomery, 2011: Inner-core vacillation cycles during the intensification of Hurricane Katrina. Quart. J. Roy. Meteor. Soc., 137, 829-844, https://doi.org/10.1002/qj.823.

Pan, Y., K. Zhu, M. Xue, X. Wang, M. Hu, S. G. Benjamin, S. S. Weygandt, and J. S. Whitaker, 2014: A GSI-based coupled EnSRFEn3DVar hybrid data assimilation system for the operational Rapid Refresh model: Tests at a reduced resolution. Mon. Wea. Rev., 142, 3756-3780, https://doi.org/10.1175/MWR-D-13-00242.1.

Persing, J., and M. T. Montgomery, 2003: Hurricane superintensity. J. Atmos. Sci., 60, 2349-2371, https://doi.org/10.1175/ 1520-0469(2003)060<2349:HS>2.0.CO;2.

,-- J. C. McWilliams, and R. K. Smith, 2013: Asymmetric and axisymmetric dynamics of tropical cyclones. Atmos. Chem. Phys., 13, 12 299-12 341, https://doi.org/10.5194/acp-1312299-2013.

$\mathrm{Pu}, \mathrm{Z}$., and S. A. Braun, 2001: Evaluation of bogus vortex techniques using four-dimensional variational data assimilation. Mon. Wea. Rev., 129, 2023-2039, https://doi.org/10.1175/1520-0493(2001) 129<2023:EOBVTW >2.0.CO;2.

- X. Li, and J. Sun, 2009: Impact of airborne Doppler radar data assimilation on the numerical simulation of intensity changes of Hurricane Dennis near a landfall. J. Atmos. Sci., 66, 3351-3365, https://doi.org/10.1175/2009JAS3121.1.

- , S. Zhang, M. Tong, and V. Tallapragada, 2016: Influence of the self-consistent regional ensemble background error covariance on hurricane inner-core data assimilation with the GSI-based hybrid system for HWRF. J. Atmos. Sci., 73, 49114925, https://doi.org/10.1175/JAS-D-16-0017.1. 
Qin, N., and D.-L. Zhang, 2018: On the extraordinary intensification of Hurricane Patricia (2015). Part I: Numerical experiments. Wea. Forecasting, 33, 1205-1224, https://doi.org/ 10.1175/WAF-D-18-0045.1.

Reasor, P. D., M. T. Montgomery, F. D. Marks, and J. F. Gamache, 2000: Low-wavenumber structure and evolution of the hurricane inner core observed by airborne dual-Doppler radar. Mon. Wea. Rev., 128, 1653-1680, https://doi.org/10.1175/1520-0493(2000) $128<1653:$ LWSAEO $>2.0 . \mathrm{CO} ; 2$.

,$- \ldots$, and L. D. Grasso, 2004: A new look at the problem of tropical cyclones in vertical shear flow: Vortex resiliency. J. Atmos. Sci., 61, 3-22, https://doi.org/10.1175/1520-0469(2004) 061<0003:ANLATP $>2.0 . \mathrm{CO} ; 2$.

Rogers, R., and Coauthors, 2013: NOAA'S Hurricane Intensity Forecasting Experiment: A progress report. Bull. Amer. Meteor. Soc., 94, 859-882, https://doi.org/10.1175/BAMS-D-12-00089.1.

— The extraordinary intensification of Hurricane Patricia (2015). Bull. Amer. Meteor. Soc., 98, 2091-2112, https:// doi.org/10.1175/BAMS-D-16-0039.1.

—_, P. D. Reasor, and J. A. Zhang, 2015: Multiscale structure and evolution of Hurricane Earl (2010) during rapid intensification. Mon. Wea. Rev., 143, 536-562, https://doi.org/ 10.1175/MWR-D-14-00175.1.

Rotunno, R., and G. H. Bryan, 2012: Effects of parameterized diffusion on simulated hurricanes. J. Atmos. Sci., 69, 22842299, https://doi.org/10.1175/JAS-D-11-0204.1.

Schubert, W. H., M. T. Montgomery, R. K. Taft, T. A. Guinn, S. R. Fulton, J. P. Kossin, and J. P. Edwards, 1999: Polygonal eyewalls, asymmetric eye contraction, and potential vorticity mixing in hurricanes. J. Atmos. Sci., 56, 1197-1223, https://doi.org/10.1175/ 1520-0469(1999)056<1197:PEAECA > 2.0.CO;2.

Schwartz, C. S., Z. Liu, X.-Y. Huang, Y.-H. Kuo, and C.-T. Fong, 2013: Comparing limited-area 3DVAR and hybrid variationalensemble data assimilation methods for typhoon track forecasts: Sensitivity to outer loops and vortex relocation. Mon. Wea. Rev., 141, 4350-4372, https://doi.org/10.1175/MWR-D-13-00028.1.

Smith, R. K., M. T. Montgomery, and S. Vogl, 2008: A critique of Emanuel's hurricane model and potential intensity theory. Quart. J. Roy. Meteor. Soc., 134, 551-561, https://doi.org/ 10.1002/qj.241.

— M. T. Montgomeryb, and N. Van Sang, 2009: Tropical cyclone spin-up revisited. Quart. J. Roy. Meteor. Soc., 135, 13211335, https://doi.org/10.1002/qj.428.

Stern, D. P., and D. S. Nolan, 2011: On the vertical decay rate of the maximum tangential winds in tropical cyclones. J. Atmos. Sci., 68, 2073-2094, https://doi.org/10.1175/2011JAS3682.1.

_ J. R. Brisbois, and D. S. Nolan, 2014: An expanded dataset of hurricane eyewall sizes and slopes. J. Atmos. Sci., 71, 27472762, https://doi.org/10.1175/JAS-D-13-0302.1.

— I. D. Doyle, G. H. Bryan, and J. D. Jepert, 2017: Understanding atypical mid-level wind speed maxima in hurricane eyewalls. 28th Conf. on Weather Analysis and Forecasting/24th Conf. on Numerical Weather Prediction, Seattle, WA, Amer. Meteor. Soc., 118, https://ams.confex. com/ams/97Annual/webprogram/Paper315144.html.

Susca-Lopata, G., J. Zawislak, E. J. Zipser, and R. F. Rogers, 2015: The role of observed environmental conditions and precipitation evolution in the rapid intensification of Hurricane Earl (2010). Mon. Wea. Rev., 143, 2207-2223, https:// doi.org/10.1175/MWR-D-14-00283.1.

Tallapragada, V., and Coauthors, 2014: Hurricane Weather Research and Forecasting (HWRF) model: 2014 scientific documentation. Developmental Testbed Center, 105 pp., https://dtcenter.org/HurrWRF/users/docs/scientific_documents/ HWRFv3.6a_ScientificDoc.pdf.

— , and Coauthors, 2015: Hurricane Weather Research and Forecasting (HWRF) model: 2015 scientific documentation. Developmental Testbed Center, 113 pp., https://dtcenter.org/ HurrWRF/users/docs/scientific_documents/HWRF_v3.7a_SD.pdf.

Thu, T. V., and T. N. Krishnamurti, 1992: Vortex initialization for typhoon track prediction. Meteor. Atmos. Phys., 47, 117-126, https://doi.org/10.1007/BF01025612.

Tong, M., and Coauthors, 2018: Impact of assimilating aircraft reconnaissance observations on tropical cyclone initialization and prediction using operational HWRF and GSI ensemblevariational hybrid data assimilation. Mon. Wea. Rev., 146, 4155-4177, https://doi.org/10.1175/MWR-D-17-0380.1.

Torn, R. D., and G. J. Hakim, 2009: Ensemble data assimilation applied to RAINEX observations of Hurricane Katrina (2005). Mon. Wea. Rev., 137, 2817-2829, https://doi.org/ 10.1175/2009MWR2656.1.

Van Sang, N. V., R. K. Smith, and M. T. Montgomery, 2008: Tropical-cyclone intensification and predictability in three dimensions. Quart. J. Roy. Meteor. Soc., 134, 563-582, https:// doi.org/10.1002/qj.235.

Velden, C., W. E. Lewis, W. Bresky, D. Stettner, J. Daniels, and S. Wanzong, 2017: Assimilation of high-resolution satellitederived atmospheric motion vectors: Impact on HWRF forecasts of tropical cyclone track and intensity. Mon. Wea. Rev., 145, 11071125, https://doi.org/10.1175/MWR-D-16-0229.1.

Vukicevic, T., A. Aksoy, P. Reasor, S. D. Aberson, K. J. Sellwood, and F. Marks, 2013: Joint impact of forecast tendency and state error biases in ensemble Kalman filter data assimilation of inner-core tropical cyclone observations. Mon. Wea. Rev., 141, 2992-3006, https://doi.org/10.1175/MWR-D-12-00211.1.

Wang, X., 2010: Incorporating ensemble covariance in the gridpoint statistical interpolation variational minimization: A mathematical framework. Mon. Wea. Rev., 138, 2990-2995, https://doi.org/10.1175/2010MWR3245.1.

— , and T. Lei, 2014: GSI-based four-dimensional ensemblevariational (4DEnsVar) data assimilation: formulation and single-resolution experiments with real data for NCEP Global Forecast System. Mon. Wea. Rev., 142, 3303-3325, https://doi.org/ 10.1175/MWR-D-13-00303.1.

— T. M. Hamill, J. S. Whitaker, and C. H. Bishop, 2007: A comparison of hybrid ensemble transform Kalman filteroptimum interpolation and ensemble square root filter analysis schemes. Mon. Wea. Rev., 135, 1055-1076, https://doi.org/ 10.1175/MWR3307.1.

—, D. M. Barker, C. Snyder, and T. M. Hamill, 2008: A hybrid ETKF-3DVAR data assimilation scheme for the WRF Model. Part II: Real observation experiments. Mon. Wea. Rev., 136, 5132-5147, https://doi.org/10.1175/2008MWR2445.1.

_ D. D. Parrish, D. Kleist, and J. Whitaker, 2013: GSI 3DVar-based ensemble-variational hybrid data assimilation for NCEP global forecast system: Single-resolution experiments. Mon. Wea. Rev., 141, 4098-4117, https://doi.org/10.1175/MWR-D-12-00141.1.

Weng, Y., and F. Zhang, 2012: Assimilating airborne Doppler radar observations with an ensemble Kalman filter for convection-permitting hurricane initialization and prediction: Katrina (2005). Mon. Wea. Rev., 140, 841-859, https://doi.org/ 10.1175/2011MWR3602.1.

Willoughby, H. E., H.-L. Jin, S. J. Lord, and J. M. Piotrowicz, 1984: Hurricane structure and evolution as simulated by an axisymmetric, nonhydrostatic numerical model. J. Atmos. Sci., 41, 
1169-1186, https://doi.org/10.1175/1520-0469(1984)041<1169: HSAEAS $>2.0 . \mathrm{CO} ; 2$.

Xiao, Q., X. Zhang, C. Davis, J. Tuttle, G. Holland, and P. J. Fitzpatrick, 2009: Experiments of hurricane initialization with airborne Doppler radar data for the Advanced Research Hurricane WRF (AHW) Model. Mon. Wea. Rev., 137, 27582777, https://doi.org/10.1175/2009MWR2828.1.

Yang, C., Z. Liu, J. Bresch, S. R. H. Rizvi, X.-Y. Huang, and J. Min, 2016: AMSR2 all-sky radiance assimilation and its impact on the analysis and forecast of Hurricane Sandy with a limited-area data assimilation system. Tellus, 68A, 30917, https://doi.org/10.3402/ tellusa.v68.30917.

Zhang, F., Y. Weng, J. A. Sippel, Z. Meng, and C. H. Bishop, 2009: Cloud-resolving hurricane initialization and prediction through assimilation of Doppler radar observations with an ensemble Kalman filter. Mon. Wea. Rev., 137, 2105-2125, https://oi.org/ 10.1175/2009MWR2645.1

,-- , J. F. Gamache, and F. D. Marks, 2011: Performance of convection-permitting hurricane initialization and prediction during 2008-2010 with ensemble data assimilation of innercore airborne Doppler radar observations. Geophys. Res. Lett., 38, L15810, https://doi.org/10.1029/2011GL048469.

—, M. Minamide, and E. E. Clothiaux, 2016: Potential impacts of assimilating all-sky infrared satellite radiances from GOES-R on convection-permitting analysis and prediction of tropical cyclones. Geophys. Res. Lett., 43, 2954-2963, https://doi.org/ 10.1002/2016GL068468.

Zhang, J. A., and M. T. Montgomery, 2012: Observational estimates of the horizontal eddy diffusivity and mixing length in the low-level region of intense hurricanes. J. Atmos. Sci., 69, 1306-1316, https://doi.org/10.1175/JAS-D-11-0180.1.

, and F. D. Marks, 2015: Effects of horizontal diffusion on tropical cyclone intensity change and structure in idealized threedimensional numerical simulations. Mon. Wea. Rev., 143, 39813995, https://doi.org/10.1175/MWR-D-14-00341.1.
D. S. Nolan, R. F. Rogers, and V. Tallapragada, 2015: Evaluating the impact of improvements in the boundary layer parameterization on hurricane intensity and structure forecasts in HWRF. Mon. Wea. Rev., 143, 3136-3155, https://doi.org/ 10.1175/MWR-D-14-00339.1.

—_, F. D. Marks, J. A. Sippel, R. F. Rogers, X. Zhang, S. G. Gopalakrishnan, Z. Zhang, and V. Tallapragada, 2018: Evaluating the impact of improvement in the horizontal diffusion parameterization on hurricane prediction in the operational Hurricane Weather Research and Forecast (HWRF) Model. Wea. Forecasting, 33, 317-329, https:// doi.org/10.1175/WAF-D-17-0097.1.

Zhou, C., H. Shao, and L. Bernardet, 2015a: Regional applications of the GSI-hybrid data assimilation for highresolution tropical storm forecasts: Tackling the intensity spin-down issue in 2014 HWRF. 16th WRF Users Workshop, Boulder, CO, Developmental Testbed Center, https:// dtcenter.org/eval/data_assim/publications/GSI-Hybrid\%20at \%202015\%20WRFUsersWorkshop.v2_poster.pdf].

, — - L. R. Bernardet, M. Tong, and V. Tallapragada, 2015b: Regional applications of the GSI-hybrid data assimilation for high-resolution tropical storm forecasts. 19th Conf. on Integrated Observing and Assimilation Systems for the Atmosphere, Oceans, and Land Surface (IOAS-AOLS), Phoenix, AZ, Amer. Meteor. Soc., 6.3, https://ams.confex.com/ams/ 95Annual/webprogram/Paper269008.html

Zhu, P., K. Menelaou, and Z. Zhu, 2014: Impact of subgrid-scale vertical turbulent mixing on eyewall asymmetric structures and mesovortices of hurricanes. Quart. J. Roy. Meteor. Soc., 140, 416-438, https://doi.org/10.1002/qj.2147.

_- B. Tyner, J. A. Zhang, E. Aligo, S. Gopalakrishnan, F. D. Marks, A. Mehra, and V. Tallapragada, 2018: Role of eyewall and rainband eddy forcing in tropical cyclone intensification. Atmos. Chem. Phys. Discuss., https://doi.org/ 10.5194/acp-2018-610, in press. 